\title{
Multiprincipals multiagents incentive design
}

\author{
Rudolf Kerschbamer ${ }^{1}$, Semih Koray ${ }^{2}$ \\ ${ }^{1}$ Department of Economics, University of Vienna, Hohenstaufengasse 9, 1010 Vienna, Austria; and \\ CEPR, London, United Kingdom (e-mail: Rudolf.Kerschbamer@univie.ac.at) \\ 2 Department of Economics, Bilkent University, 06533 Bilkent, Ankara, Turkey \\ (e-mail: ksemih@bilkent.edu.tr)
}

Received: 14 June 1995 / Accepted: 09 August 1999

\begin{abstract}
This paper studies a simple setting in which the contractual arrangements which determine the incentives for agents are not designed by a single central planner, but are themselves the outcome of a game among multiple noncooperatively acting principals. The notion of an Epsilon Contracting Equilibrium is introduced to predict the outcome of the contract-design game among principals. Symmetric pure strategy Epsilon Contracting Equlibria may not exist in perfectly symmetric environments. In a symmetric Epsilon Contracting Equilibrium in mixed strategies coordination failure may lead to a suboptimal institutional network in which the agents "cheat" their principals.
\end{abstract}

JEL classification: C72, D82

Key words: Adverse selection, multiprincipals, multiagents, epsilon contracting equilibrium

\section{Introduction}

A large part of the theoretical literature on agency problems concentrates on the question of how a relatively uninformed individual (the principal) should use her power to design both the rules of communication and the structure of incentives for a group of other individuals (the agents), so as to maximize her own expected payoff. Depending on the specification of the feasible outcomes, the agents' preferences and information, the imputed game-theoretic solution concept and the objectives of the principal, different basic models have been

This paper was previously titled "Coordination Failures in the Design of Incentives". Thanks for useful comments are due to two anonymous referees and to seminar audiences at the University of Vienna and at Northwestern University. Of course, the usual disclaimer applies. 
applied to a great variety of contexts. Indeed, one of the merits of the principalagent paradigm has been to show that such diverse relationships as those between an employer and her employees, between an auctioneer and a set of buyers, between a governmental agency and the relevant residents, between a regulator and a fringe of regulated firms, between a monopolist and her potential customers can all be handled within the same theoretical framework. On the other hand, the standard version of the principal-agent model, as it stands, may not provide an adequate description for some of these relationships in which case it needs to be modified appropriately, of course.

In some applications, there is indeed a single individual who has the power to choose the rules which maximize her expected utility, when the reaction correspondences of agents derived from their objective functions and the imputed equilibrium concept are given. In other applications, there is no single central planner, but one can imagine ex ante negotiations between uninformed players to have lead to an outcome which looks as if it were designed by a single individual with well defined goals. ${ }^{1}$ It is not very rare, however, that the standard approach to mechanism design is applied to situations in which it is not clear at all why the institutional arrangements which determine the incentives for a given set of players should exhibit the same features as those derived under the single-planner assumption. One can cite the literature on relative performance evaluation and remuneration for top managers in large publicly held corporations as an example for such an application. Virtually all of the theoretical literature concerned with this issue works under the single-planner assumption. The formal results derived from the model under this assumption are then applied to situations in which the incentives for different agents are determined by different principals through heuristic arguments.

This paper is a deviation from the single-planner paradigm. It studies a simple model in which the institutional arrangements which determine the incentives for agents are themselves the outcome of a simultaneous-move game in which two principals interact in choosing contracts. More specifically, a two-stage game is formulated, in the first stage of which each principal designs a contract for her own agent(s). In the second stage, the agents, having observed the contracts committed to in the first stage, play a Bayesian game.

The equilibrium notion we employ in predicting the outcome in the first stage of this two-stage game is Epsilon Contracting Equilibrium. Taking Radner's (1980) epsilon equilibrium as the main building block, we introduce a new notion of equilibrium which can roughly be described as a continuum of epsilon equilibria which approximate a state of equilibrium as closely as one wishes, without ever actually reaching it when it is absent. Whenever a Nash equilibrium exists in the contract-choosing game among principals, on the other hand, our Epsilon Contracting Equilibrium (henceforth ECE) simply reduces to that equilibrium. The need for an ECE arises in our context from the conjunction of the discrete nature of the type spaces of the agents with the continuous na-

\footnotetext{
${ }^{1}$ One might, for example, imagine that the members of a committee agree on a set of rules of behavior before they receive their private information.
} 
ture of admissible contract spaces. The dependence of agents' induced behaviors upon contract combinations exhibits jump discontinuities which, in turn, lead to discontinuities of the same kind in the principals' utilities. Moreover, natural candidates of contract combinations for equilibria are to be sought on the boundaries separating regions which correspond to different induced behaviors on the part of agents, for the principals not only wish to induce their agents to behave in a particular fashion, but they also wish to achieve this aim as cheaply as possible. In our present model, some focal contract combinations thus turn out not to be Nash equilibria, but cluster points of epsilon equilibria getting arbitrarily close to a state of equilibrium. This main reason for employing the notion of ECE also forms a first major difference of the present paper from earlier studies which use standard Nash equilibrium to analyze interactions among several principals.

Another difference concerns the way the problem of multiple equilibria in the agents' game is dealt with. Many authors restrict their attention to incentivecompatible contracts only and thus ignore the problem of multiple equilibria. This amounts to assuming that the agents coordinate their expectations on the principals' preferred solution in each case. In contrast to this, an explicit set of equilibrium selection criteria is employed in the present paper to predict the outcome in the agents' game. A final difference is concerned with the observability of contracts. Some authors assume that contracts are both observable and verifiable, so that contract-contingent contracts are feasible. This assumption inevitably leads to a highly cooperative outcome in the game among principals. Others suppose that contracts are private information to the parties who signed the contract. This prevents the agents from conditioning their strategies on the overall institutional network. The scenario studied here is somewhere inbetween: The agents are able to observe the contractual network when upon to move; contract-contingent contracts are, however, infeasible because each contract is verifiable only by the principal-agent pair who signed it.

The particular agency problem studied here can be summarized as follows: There are two principals, each hiring a single agent. The outcome in each principal-agent hierarchy is entirely determined by the action taken by the agent in that pair along with the realization of a random variable for that hierarchy, and it is independent of what happens in the other hierarchy. That is, there is no "market interdependence" between the two principal-agent pairs. There is, however, an interdependence between the informational structures of the two hierarchies since the random variables for these are assumed to be positively but imperfectly correlated. An incentive problem arises because both the realization of the random variable and the outcome in each principal-agent pair are privately observed by the agent in that hierachy.

An agent's utility depends solely on his share of the resulting profit, and there is no disutility associated with any particular decision. An agent's incentive to misrepresent his private information originates from the fact that any part of the profit that is not paid out to the principal can be kept by the agent. Principals deal with this incentive problem by designing contracts through which the payouts each agent is supposed to make to his principal are specified. Since agents' 
decisions are publicly observable but profits are not, an agent's payout can depend both on his and the other agent's decisions, but not on profits in either hierarchy.

Contracts which allow the payouts to also depend upon the other agent's decisions are clearly superior to those in which the payouts are functions of only one's own agent's decisions from the viewpoint of the principals. Through the former kind of contracts ("relative performance contracts") the principal can indirectly get some information (though imperfect) about the realized value of the random variable observed by her own agent. This information is valuable to her because she can use it to reduce her agent's informational rent.

Committing to the cheapest relative performance contract is risky: If both agents operate under such a contract they can cheat their principals by jointly adopting strategies not intended for them. To avoid this, at least one principal has to choose a more powerful contract which, however, causes additional costs. This generates a sort of public good problem in the contract design game among principals.

It is shown that, in spite of the symmetric structure of the principals' game, there are no symmetric pure strategy Epsilon Contracting Equlibria mainly due to the fact that one powerful contract is not only necessary, but also sufficient to induce both agents to exhibit the desired behavior. On the other hand, a symmetric ECE in mixed strategies exists, leading to suboptimal institutional arrangements with positive probability due to coordination failures among the principals. Although the institutional arrangements resulting from the symmetric ECE in mixed strategies are not efficient relative to incentive constraints, an improvement is still achieved upon the situation where the principals restrict themselves to independent contracts without taking advantage of the existing informational interdependence.

The plan of the paper is as follows: The model is presented in the next section. Section 3 introduces the notion of an ECE. Section 4 presents the results. Some related work is discussed in Section 5, and Section 6 concludes. $^{2}$

\section{The model}

\subsection{Technologies}

We consider two ex ante identical hierarchies, indexed by $i=\alpha, \beta$. Each hierarchy ("firm") $i$ is owned by a single principal $P^{i}$ and run by a single agent $A^{i}$. Each $A^{i}$ 's task is to make a decision $d^{i}$ from a decision set $D^{i}$. $A^{i}$, s decision together with the realization of a random variable $\theta^{i} \in \Theta^{i}$ determines the outcome ("profit") $x^{i}$ for firm $i$ according to the commonly known relationship (identical for both firms) $x^{i}=\phi\left(\theta^{i}, d^{i}\right)$, where, for all $d^{i} \in D^{i}$ and $\theta^{i} \in \Theta^{i}$,

\footnotetext{
2 Before proceeding any further, we would like to acknowledge earlier work on problems of multiple equilibria in principal-agent models as presented, for example, by Mookherjee (1984), Demski and Sappington (1984), Ma et al. (1988) and Kerschbamer (1994). Although none of these papers considers strategic interactions among multiple principals (and therefore none of them is able to explain the appearance of institutional arrangements which fail to implement the desired outcome) the present work nevertheless makes considerable use of their insights.
} 


$$
\phi\left(\theta^{i}, d^{i}\right)>0 .
$$

The two random variables $\theta^{\alpha}$ and $\theta^{\beta}$ are drawn from a symmetric joint distribution $r(\cdot)$ on $\Theta^{\alpha} \times \Theta^{\beta}$, where $\Theta^{\alpha}=\Theta^{\beta}$. We assume binary support for these random variables, i.e., $\Theta^{i}=\left\{\theta_{1}^{i}, \theta_{2}^{i}\right\}$ with $\theta_{1}^{i} \neq \theta_{2}^{i}$. The realization of $\theta_{2}^{i}$ is assumed to imply higher profits than that of $\theta_{1}^{i}$ for each of $A^{i}$,s possible decisions; in other words, for each $d^{i} \in D^{i}$,

$$
\phi\left(\theta_{2}^{i}, d^{i}\right)>\phi\left(\theta_{1}^{i}, d^{i}\right)
$$

The agents' decision sets $D^{\alpha}$ and $D^{\beta}$ are assumed to be binary $\left(D^{i}=\left\{d_{1}^{i}, d_{2}^{i}\right\}\right)$ and identical $\left(D^{\alpha}=D^{\beta}\right)$. For each realization of $\theta^{i}$ a different decision $d^{i} \in D^{i}$ is the better choice; more specifically, whenever $\{k, l\}=\{1,2\}$,

$$
\phi\left(\theta_{k}^{i}, d_{k}^{i}\right)>\phi\left(\theta_{k}^{i}, d_{l}^{i}\right) .
$$

The random variables $\theta^{\alpha}$ and $\theta^{\beta}$ are positively but imperfectly correlated. That is, defining $r_{k l}^{\alpha} \equiv r\left(\theta_{k}, \theta_{l}\right)$ and $r_{k l}^{\beta} \equiv r\left(\theta_{l}, \theta_{k}\right)$ it is assumed that, for each $i \in\{\alpha, \beta\}$,

$$
1>r_{11}^{i} /\left(r_{11}^{i}+r_{12}^{i}\right)>r_{21}^{i} /\left(r_{21}^{i}+r_{22}^{i}\right)>0 .
$$

To simplify the exposition, let $R_{k l}^{i}=r_{k l}^{i} /\left(r_{k 1}^{i}+r_{k 2}^{i}\right)(k, l \in\{1,2\}, i \in\{\alpha, \beta\})$. We will refer to (1)-(4) above as ASsumptions (1)-(4) in the sequel.

\subsection{Time and information structure}

The binary supports of the random variables, the binary decision sets of the agents and the profit functions of the firms are assumed to be common knowledge to all the parties involved and all share the same prior $r(\cdot)$ on $\Theta^{\alpha} \times \Theta^{\beta}$. At Stage 1, each principal $P^{i}$ (she) offers a contract to her agent $A^{i}$. The principals make their contract proposals simultaneously and noncooperatively. The contracts then become public knowledge, and each agent $A^{i}$ (he) either accepts or rejects the contract offered by his principal $P^{i}$ after having acquired perfect information about the realization of the random variable for his firm. If $A^{i}$ rejects, firm $i$ disappears. If he accepts, the contract becomes binding. At Stage 2 the agents who have accepted their contracts move simultaneously in making their decisions $d^{\alpha}$ and $d^{\beta}$ which then become publicly observable and verifiable. The profit of each firm is privately observed by the agent of that firm.

\subsection{Contracts}

Let $C^{i}$ denote $P^{i}$,s decision domain, i.e. the set of contracts she can offer to her agent $A^{i}$. Each contract $c^{i} \in C^{i}$ consists of a "decision recommendation" $f^{i}: \Theta^{i} \rightarrow D^{i}$ and a payout schedule $g^{i}(\cdot)$. The decision recommendation $f^{i}(\cdot)$ specifies the actions principal $P^{i}$ desires her agent $A^{i}$ to choose as a function of the values the random variable $\theta^{i}$ may take, and it is also used as a "tie-breaking 
rule" by the agents when indifferences arise. The payout schedule $g^{i}(\cdot)$ specifies a contingent transfer from $A^{i}$ to $P^{i}$. In order to be enforceable the payout schedule must be conditioned on verifiable variables. The only verifiable variables in the present model are the agents' Stage 2 decisions $d^{\alpha}$ and $d^{\beta}$ along with the contract-acceptance-rejection decisions in Stage $1 .^{3}$ So each payout schedule is a function $g^{i}: D^{i} \times D^{j} \rightarrow \mathbf{R}$, where $\{i, j\}=\{\alpha, \beta\} .^{4}$ If we denote $f_{h}^{i} \equiv f^{i}\left(\theta_{h}^{i}\right)$ and $g_{k l}^{i} \equiv g^{i}\left(d_{k}^{i}, d_{l}^{j}\right)$, we can represent each contract by a vector

$$
c^{i}=\left(f_{1}^{i}, f_{2}^{i}, g_{11}^{i}, g_{12}^{i}, g_{21}^{i}, g_{22}^{i}\right) .
$$

We let $C$ (with typical element $c$ ) denote the set of all contract combinations $C^{\alpha} \times C^{\beta}$. The set of all combinations of Stage 2 decisions $D^{\alpha} \times D^{\beta}$ is denoted $D$, with typical element $d$.

\subsection{Preferences}

The principals are assumed to be risk neutral. Their objective is to maximize expected payouts. The two agents are identical regarding their preferences as well. Their twice continuously differentiable, strictly concave, strictly increasing, von Neumann-Morgenstern utility functions $U(\cdot)$ for money exhibit nonincreasing absolute risk aversion (NIARA). ${ }^{5}$ Agents' utility functions are common knowledge as is the magnitude of their reservation utility $\bar{U}$. Both the reservation utility and the amount of money required to guarantee the reservation utility are normalized to equal zero: $U(0)=\bar{U}=0$.

\subsection{Payoffs}

First consider the case where both agents have accepted the contracts offered in Stage 1. Then for a given vector of contracts $c=\left(\hat{c}^{\alpha}, \hat{c}^{\beta}\right)$ with $\hat{c}^{i}=\left(\hat{f}_{1}^{i}, \hat{f}_{2}^{i}\right.$, $\left.\hat{g}_{11}^{i}, \hat{g}_{12}^{i}, \hat{g}_{21}^{i}, \hat{g}_{22}^{i}\right)$, a given "environment" $\theta=\left(\theta_{j}^{\alpha}, \theta_{h}^{\beta}\right)$, and a given action profile $d=\left(d_{k}^{\alpha}, d_{l}^{\beta}\right)$, the payoffs for $P^{i}\left(\right.$ denoted $\left.p^{i}\right)$ and $A^{i}$ (denoted $\left.a^{i}\right)$ are $p^{i}(c, d, \theta)=$ $\hat{g}_{k l}^{i}$ and $a^{i}(c, d, \theta)=U\left(\phi\left(\theta_{j}^{i}, d_{k}^{i}\right)-\hat{g}_{k l}^{i}\right)$ for $i \in\{\alpha, \beta\}$. The principals' and agents' payoffs for the case where at least one firm gets closed down are obtained in the obvious manner.

\footnotetext{
${ }^{3}$ Since situations in which one of the firms is closed down are of limited interest, we deal with the agents' acceptance-rejection decisions in a fairly rudimentary way (see the next footnote and Appendix 1).

${ }^{4}$ A payout schedule of this form is not wholly comprehensive, for it does not specify what the transfer from $A^{i}$ to $P^{i}$ should be in case the other firm gets closed down. We assume that each contract contains a small clause specifying that the amount of resources $A^{i}$ has to disburse to $P^{i}$ in that case is $g_{1}^{i} \equiv g^{i}\left(d_{1}^{i},-\right)=\phi\left(\theta_{1}, d_{1}\right) ; g_{2}^{i} \equiv g^{i}\left(d_{2}^{i},-\right)=\phi\left(\theta_{1}, d_{1}\right)+\phi\left(\theta_{2}, d_{2}\right)-\phi\left(\theta_{2}, d_{1}\right)$. Although the specification of this amount is part of the contract design and thus should be left to the principals, we fix it as above once and for all since it represents the unique level of transfer arising from optimizing behavior of the parties involved, as will be shown in Lemma 1 below.

5 This assumption facilitates the analysis by ensuring that randomized payout schedules are strictly dominated by deterministic ones.
} 


\section{Definition of equilibrium}

We employ the notion of an Epsilon Contracting Equilibrium (ECE) to analyze the contract-design game among principals. The definition of ECE is in the spirit of Perfect Equilibrium in the sense that we begin by analyzing the last stage of the game which we refer to as "the agents' game" and whose resolution yields the payoffs the principals will receive in their own game. The principals' contract proposals conjoined with the agents' acceptance decisions upon having observed the realizations of the random variable $\theta$ for their own firms lead to the agents' game, where the natural equilibrium concept to be employed is that of a Bayesian Equilibrium (BE), for $A^{i}$ cannot observe $\theta^{j}$ (where $\{i, j\}=\{\alpha, \beta\}$ ), but his prior about $\theta^{j}$ coincides with that of $A^{j}$ about $\theta^{i}$. Below we will give the definition of a pure strategy $\mathrm{BE}$ for the case where both agents accept the contracts offered under both realizations of $\theta$ for their own firms. Each $A^{i}$, s pure strategy set in this game thus consists of functions $e^{i}: \Theta^{i} \rightarrow D^{i}$. The extension of the equilibrium notion here to mixed strategies as well as to agents' games where some agents reject the contract they are offered under some relization of $\theta$ is straightforward and thus omitted.

Definition. A strategy profile $\bar{e}(\cdot)=\left(\bar{e}^{\alpha}(\cdot), \bar{e}^{\beta}(\cdot)\right)$ is a pure strategy Bayesian Equilibrium in the agents' game generated by an accepted contract combination $\hat{c} \in C$ if, for every $i \in\{\alpha, \beta\}$ and for every $\theta_{h}^{i} \in \Theta^{i}, e^{i}\left(\theta_{h}^{i}\right)=\bar{e}^{i}\left(\theta_{h}^{i}\right)$ maximizes $R_{h 1}^{i} a^{i}\left(\hat{c}^{i}, e^{i}\left(\theta_{h}^{i}\right), \bar{e}^{j}\left(\theta_{1}^{j}\right), \theta_{h}^{i}\right)+R_{h 2}^{i} a^{i}\left(\hat{c}^{i}, e^{i}\left(\theta_{h}^{i}\right), \bar{e}^{j}\left(\theta_{2}^{j}\right), \theta_{h}^{i}\right)$.

Let $\Phi(c)$ denote the set of all (mixed-strategy) Bayesian Equilibria induced by an accepted $c \in C$. Since the agents' game is finite, there exists at least one $\mathrm{BE}$ for each $c$ so that $\# \Phi(c) \geq 1$. If $\# \Phi(c)>1$ for a given $c$ in $C$, we use a set of equilibrium selection criteria to choose a single element $\bar{e}(\cdot)$ in $\Phi(c)$. In other words, we assume that the agents resolve their game according to a refinement of the BE concept which will be specified below and turn out to be singleton-valued for all relevant agents' games here. The selection criteria employed are Weak Firm Loyalty (WFL) ${ }^{6}$ and Payoff Dominance

6 WFL consists of two subcriteria denoted by $\mathrm{WFL}_{0}$ and $\mathrm{WFL}_{1}$. WFL 0 pertains to the acceptancerejection decisions of the offered contracts in Stage 1 , whereas $\mathrm{WFL}_{1}$ is applied to the agents' game in Stage 2. WFL 1 for firm $\alpha\left(\mathrm{WFL}_{1}^{\alpha}\right)$ is defined as follows: "Take any pair $\hat{c}=\left(\hat{c}^{\alpha}, \hat{c}^{\beta}\right)$ of contracts with $\# \Phi(\hat{c})>1$. Note that the decision recommendation pair $\hat{f}=\left(\hat{f}^{\alpha}, \hat{f}^{\beta}\right)$ in $\hat{c}$ is a strategy profile in the agents' game. If $\hat{f} \in \Phi(\hat{c})$ and $\tilde{e}=\left(\tilde{e}^{\alpha}, \tilde{e}^{\beta}\right) \in \Phi(\hat{c})$ with $\tilde{e}^{\alpha} \neq \hat{f}^{\alpha}$ is such that the interim expected utility of $A^{\alpha}$ for each realization of $\theta^{\alpha}$ is the same under $\hat{f}$ and $\tilde{e}$, then $\tilde{e}$ is eliminated from $\Phi(\hat{c})$." WFL ${ }_{1}^{\alpha}$ and its mirror image $\mathrm{WFL}_{1}^{\beta}$ are applied sequentially, beginning with $\mathrm{WFL}_{1}^{\alpha}$. WFL 0 for firm $\alpha\left(\mathrm{WFL}_{0}^{\alpha}\right)$ is defined as follows: "Given any pair $\hat{c}=\left(\hat{c}^{\alpha}, \hat{c}^{\beta}\right)$ of contracts for which the $\mathrm{WFL}_{1}^{\alpha}$-refinement of $\Phi(\hat{c})$ is a singleton, if the interim expected utility of $A^{\alpha}$ under the particular realization of $\theta^{\alpha}$ which he observes is $\bar{U}(=0)$, then he accepts $\hat{c}^{\alpha}$." WFL ${ }_{0}^{\alpha}$ and its mirror image $\mathrm{WFL}_{0}^{\beta}$ are again applied sequentially, beginning with $\mathrm{WFL}_{0}^{\alpha}$. Kerschbamer (1998) applies a different version of $\mathrm{WFL}_{1}$. In that version $\mathrm{WFL}_{1}^{\alpha}$ eliminates $\tilde{e}$ from $\Phi(\hat{c})$ whenever $\left(\hat{f}^{\alpha}, \tilde{e}^{\beta}\right) \in \Phi(\hat{c})$. In the present context this latter version turns out to be stronger than the one employed here as also will be noted in Footnote 22 below. 
$(\mathrm{PD})^{7}$. The reason for applying WFL is purely technical: It helps to avoid a trivial multiplicity of equilibria resulting from agents being indifferent among two or more strategies. ${ }^{8}$ We first apply WFL, and then delete the payoff dominated BE from the WFL-refinement of $\Phi$ we thus obtain, yielding the final refinement of $\Phi$ according to which the agents' game is resolved. Henceforth, we will let $\Phi(c)$ stand for the conjoined refinement. If $\Phi(c)$ is a singleton for a given $c \in C$ the interim expected utility of $A^{i}$ for each realization of $\theta^{i}$ under $c$ is given by $V_{A}^{i}\left(c \mid \theta_{h}^{i}\right)=R_{h 1}^{i} a^{i}\left(c^{i}, \bar{e}^{i}\left(\theta_{h}^{i}\right), \bar{e}^{j}\left(\theta_{1}^{j}\right), \theta_{h}^{i}\right)+R_{h 2}^{i} a^{i}\left(c^{i}, \bar{e}^{i}\left(\theta_{h}^{i}\right), \bar{e}^{j}\left(\theta_{2}^{j}\right), \theta_{h}^{i}\right)$, where $\bar{e}=\left(\bar{e}^{\alpha}, \bar{e}^{\beta}\right)$ is the unique element in $\Phi(c)$. If for all $i \in\{\alpha, \beta\}$ and $\theta_{h}^{i} \in \Theta^{i}$ we have $V_{A}^{i}\left(c \mid \theta_{h}^{i}\right) \geq 0$, then each $P^{i}$, s ex ante valuation of $c$ is given by $V_{P}^{i}\left(c^{i}, c^{j}\right)=$ $\sum_{g=1}^{2} \sum_{h=1}^{2} r_{g h}^{i} p^{i}\left(c^{i}, \bar{e}^{i}\left(\theta_{g}^{i}\right), \bar{e}^{j}\left(\theta_{h}^{j}\right)\right)$. If $V_{A}^{i}\left(c \mid \theta_{h}^{i}\right)<0$ for at least one realization of $\theta^{i}$ for at least one agent $i$, then the individual-rationality constraint for this $\theta^{i}$ realization is violated. The $V_{P}^{i}\left(c^{i}, c^{j}\right)$ 's are then defined differently. Appendix 1 addresses this issue.

Having derived $V_{P}^{i}\left(c^{i}, c^{j}\right)$ for each $c \in C$, we now can use these reduced-form payoffs in the definition of equilibrium for the contract-writing game. Although the strategy spaces in the contracting game can be chosen as compact subsets of a Euclidean space, standard noncooperative (Nash) equilibria do not necessarily exist because the structure of the game induces discontinuities in the principals' payoff functions. The equlibrium concept used to predict the outcome in the game among principals is therefore defined in the spirit of Radner's (1980) epsilonequilibrium:

Definition. Let $\epsilon \geq 0$, and $\{i, j\}=\{\alpha, \beta\}$. For any two contracts $\bar{c}^{i} \in C^{i}, \bar{c}^{j} \in$ $C^{j}, \bar{c}^{i}$ is said to be a pure strategy $\epsilon$-best response to $\bar{c}^{j}$ iff $\forall c^{i} \in C^{i}: V_{P}^{i}\left(c^{i}, \bar{c}^{j}\right) \leq$ $V_{P}^{i}\left(\bar{c}^{i}, \bar{c}^{j}\right)+\epsilon$. Moreover, $\left(\bar{c}^{\alpha}, \bar{c}^{\beta}\right) \in C^{\alpha} \times C^{\beta}$ is said to be a pure strategy $\epsilon$ equilibrium (in the contracting game among principals) iff $\forall i \in\{\alpha, \beta\}: \bar{c}^{i}$ is a pure strategy $\epsilon$-best response to $\bar{c}^{j}$.

Now let $\bar{\epsilon}_{\alpha}, \bar{\epsilon}_{\beta}>0$, and $\bar{c}^{i}:\left[0, \bar{\epsilon}_{i}\right) \rightarrow C^{i}$ with $\bar{c}^{i}(\epsilon)=\left(f_{1}^{i}, f_{2}^{i}, g_{11}^{i}(\epsilon), g_{12}^{i}(\epsilon)\right.$, $\left.g_{21}^{i}(\epsilon), g_{22}^{i}(\epsilon)\right)$ for each $\epsilon \in\left[0, \bar{\epsilon}_{i}\right)$ be such that $g_{k l}^{i}$ is continuous on $\left[0, \bar{\epsilon}_{i}\right)$ for all $i \in\{\alpha, \beta\}$ and all $k, l \in\{1,2\}$. We say that $\bar{c}^{i}$ is a pure strategy Epsilon Best Response to $\bar{c}^{j}$ iff $\forall \epsilon \in\left(0, \bar{\epsilon}_{i}\right): \bar{c}^{i}(\epsilon)$ is a pure strategy $\epsilon$-best reponse to $\bar{c}^{j}(\epsilon)$; and $\left(\bar{c}^{\alpha}, \bar{c}^{\beta}\right)$ is called a pure strategy Epsilon Contracting Equilibrium iff $\forall i \in\{\alpha, \beta\}: \bar{c}^{i}$ is a pure strategy Epsilon Best Response to $\bar{c}^{j}$.

${ }^{7} \mathrm{~A} \mathrm{BE} \bar{e} \in \Phi(c)$ payoff dominates a BE $\overline{\bar{e}} \in \Phi(c)$ iff for every $i \in\{\alpha, \beta\}$ and for every $h \in\{1,2\}: R_{h 1}^{i} a^{i}\left(c^{i}, \bar{e}^{i}\left(\theta_{h}^{i}\right), \bar{e}^{j}\left(\theta_{1}^{j}\right), \theta_{h}^{i}\right)+R_{h 2}^{i} a^{i}\left(c^{i}, \bar{e}^{i}\left(\theta_{h}^{i}\right), \bar{e}^{j}\left(\theta_{2}^{j}\right), \theta_{h}^{i}\right) \geq$ $R_{h 1}^{i} a^{i}\left(c^{i}, \overline{\bar{e}}^{i}\left(\theta_{h}^{i}\right), \overline{\bar{e}}^{j}\left(\theta_{1}^{j}\right), \theta_{h}^{i}\right)+R_{h 2}^{i} a^{i}\left(c^{i}, \overline{\bar{e}}^{i}\left(\theta_{h}^{i}\right), \overline{\bar{e}}^{j}\left(\theta_{2}^{j}\right), \theta_{h}^{i}\right)$ with $\{i, j\}=\{\alpha, \beta\}$, where the inequality is strict for at least one $h$ for each $i$.

8 The conjunction of "WFL-dominance" and PD into a lexicographic ordering on $\Phi(c)$, where WFL is taken as the primary and PD as the secondary criterion, yields a reflexive and transitive relation on $\Phi(c)$ which is not necessarily complete. The refinement of $\Phi(c)$ we work with here simply consists of the maximal elements in $\Phi(c)$ with respect to this preorder. As also noted before, although this refinement will not be singleton-valued everywhere, it will be so for all relevant contract profiles, so that it will make no difference how ties are broken in the case of thick maximal indifference classes. 
In other words, a pure strategy $\epsilon$-equilibrium is a pair of contracts such that no principal can expect to gain more than $\epsilon$ by switching to any other admissible contract, instead of playing the one specified for him. A pure strategy Epsilon Contracting Equilibrium is a pair of $\epsilon$-dependent contracts (satisfying a certain continuity requirement) such that for any $\epsilon$ from some open interval $\left(0, \bar{\epsilon}_{i}\right)$, the respective contracts form a pure strategy $\epsilon$-equilibrium. Here note that a 0 -best response is a best response in the standard sense and a 0 -equilibrium is nothing but a Nash equilibrium (in the contracting game among principals). In the sequel we will call a 0 -equilibrium simply a Contracting Equilibrium (CE).

If $\bar{c}=\left(\bar{c}^{\alpha}, \bar{c}^{\beta}\right)$ is a pure strategy Epsilon Contracting Equilibrium (ECE) and $\{i, j\}=\{\alpha, \beta\}$, then $\bar{c}^{i}(0)$ need not be a 0 -best response to $\bar{c}^{j}(0)$ and thus $\left(\bar{c}^{\alpha}(0), \bar{c}^{\beta}(0)\right)$ need not be a Nash equilibrium in the principals' contracting game although $\lim _{\epsilon \rightarrow 0} \bar{c}^{i}(\epsilon)=\bar{c}^{i}(0)$ by continuity of $\bar{c}^{i}$. In fact, this is exactly the reason why we employ the notion of an ECE rather than just simply that of a Nash equilibrium in resolving the principals' game. Here the crucial point to note is that if one confines himself to a domain of contract pairs which induce the same agents' behavior, then the principals' payoffs are continuous functions of the contract pairs, whereas the same functions exhibit jump discontinuities as one passes from one domain to another of contract pairs corresponding to different BE in the agents' game. The reason for employing the notion of an ECE is exactly the existence of contract pairs which themselves are not Nash equilibria, but are clusterpoints of a domain on which the induced agents' BE stays constant, and thus can be approached through $\epsilon$-equilibria where $\epsilon$ gets arbitrarily small.

Finally, given $c^{i} \in C^{i}$ and $\bar{c}^{j}:\left[0, \bar{\epsilon}_{j}\right) \rightarrow C^{j}(\{i, j\}=\{\alpha, \beta\})$, when one talks of $c^{i}$ being a (pure strategy) Epsilon Best Response to $\bar{c}^{j}$ or of $\bar{c}^{j}$ being a (pure strategy) Epsilon Best Response to $c^{i}$, one thinks of $c^{i}$ as being represented by $\bar{c}^{i}:\left[0, \bar{\epsilon}_{i}\right) \rightarrow C^{i}$ (for some $\bar{\epsilon}_{i}>0$ ) with $\bar{c}^{i}(\epsilon)=c^{i}$ for all $\epsilon \in\left[0, \bar{\epsilon}_{i}\right.$ ).

As usual, we allow randomization over finite sets of contracts in a mixed strategy Epsilon Contracting Equilibrium whose formal definition is given below.

Definition. Let $c_{1}^{\alpha}, \ldots, c_{k_{\alpha}}^{\alpha} \in C^{\alpha}, c_{1}^{\beta}, \ldots, c_{k_{\beta}}^{\beta} \in C^{\beta}$, let $\pi^{\alpha}:\left\{c_{1}^{\alpha}, \ldots, c_{k_{\alpha}}^{\alpha}\right\} \rightarrow$ $[0,1], \pi^{\beta}:\left\{c_{1}^{\beta}, \ldots, c_{k_{\beta}}^{\beta}\right\} \rightarrow[0,1]$ be probability distributions on their respective domains, and let $\{i, j\}=\{\alpha, \beta\}$. For any $\epsilon \geq 0$, we say that $\gamma^{i}=$ $\left(\left(c_{1}^{i}, \ldots, c_{k_{i}}^{i}\right) ; \pi^{i}\right)$ is a mixed strategy $\boldsymbol{\epsilon}$-best response to $\gamma^{j}=\left(\left(c_{1}^{j}, \ldots, c_{k_{j}}^{j}\right) ; \pi^{j}\right)$ iff, for any nonempty finite collection $\left\{c_{1}^{\prime i}, \ldots, c_{m}^{\prime}{ }^{i}\right\} \subset C^{i}$ and any probability distribution $\pi^{\prime i}$ on $\left\{c_{1}^{\prime i}, \ldots, c_{m}^{\prime i}\right\}$, one has $\sum_{t=1}^{m} \sum_{s=1}^{k_{j}} \pi^{\prime i}\left(c_{t}^{\prime i}\right) \pi^{j}\left(c_{s}^{j}\right) V_{P}^{i}\left(c_{t}^{\prime i}, c_{s}^{j}\right) \leq$ $\sum_{t=1}^{k_{i}} \sum_{s=1}^{k_{j}} \pi^{i}\left(c_{t}^{i}\right) \pi^{j}\left(c_{s}^{j}\right) V_{P}^{i}\left(c_{t}^{i}, c_{s}^{j}\right)+\epsilon$. Moreover, $\left(\gamma^{\alpha}, \gamma^{\beta}\right)$ is said to be a mixed strategy $\boldsymbol{\epsilon}$-equilibrium iff $\forall i \in\{\alpha, \beta\}: \gamma^{i}$ is a mixed strategy $\epsilon$-best response to $\gamma^{j}$.

Now let $\bar{c}_{1}^{\alpha}, \ldots, \bar{c}_{k_{\alpha}}^{\alpha}:[0, \bar{\epsilon}) \rightarrow C^{\alpha} ; \bar{c}_{1}^{\beta}, \ldots, \bar{c}_{k_{\beta}}^{\beta}:[0, \bar{\epsilon}) \rightarrow C^{\beta}$ be such that $g_{l, s t}^{i}$ is continuous on $[0, \bar{\epsilon})$, where $\bar{c}_{l}^{i}(\epsilon)=\left(f_{l, 1}^{i}, f_{l, 2}^{i}, g_{l, 11}^{i}(\epsilon), g_{l, 12}^{i}(\epsilon), g_{l, 21}^{i}(\epsilon), g_{l, 22}^{i}(\epsilon)\right)$ $(\epsilon \in[0, \bar{\epsilon}))$ for all $i \in\{\alpha, \beta\}, l \in\left\{1, \ldots, k_{i}\right\}, s, t \in\{1,2\}$. Moreover, let 
$\bar{\pi}^{i}[\epsilon]:\left\{\bar{c}_{1}^{i}(\epsilon), \ldots, \bar{c}_{k_{i}}^{i}(\epsilon)\right\} \rightarrow[0,1]$ be a probability distribution for all $\epsilon \in[0, \bar{\epsilon})$ and $i \in\{\alpha, \beta\}$. We say that $\bar{\gamma}^{i}=\left(\left(\bar{c}_{1}^{i}, \ldots, \bar{c}_{k_{i}}^{i}\right) ;\left\{\bar{\pi}^{i}[\epsilon] \mid \epsilon \in[0, \bar{\epsilon})\right\}\right)$ is a mixed strategy Epsilon Best Response to $\bar{\gamma}^{j}=\left(\left(\bar{c}_{1}^{j}, \ldots, \bar{c}_{k_{j}}^{j}\right) ;\left\{\bar{\pi}^{j}[\epsilon] \mid \epsilon \in[0, \bar{\epsilon})\right\}\right)$, iff $\bar{\gamma}^{i}(\epsilon)=\left(\left(\bar{c}_{1}^{i}(\epsilon), \ldots, \bar{c}_{k_{i}}^{i}(\epsilon)\right) ; \bar{\pi}^{i}[\epsilon]\right)$ is a mixed strategy $\epsilon$-best response to $\bar{\gamma}^{j}(\epsilon)=$ $\left(\left(\bar{c}_{1}^{j}(\epsilon), \ldots, \bar{c}_{k_{j}}^{j}(\epsilon)\right) ; \bar{\pi}^{j}[\epsilon]\right)$ for each $\epsilon \in(0, \bar{\epsilon})$. Finally, we call $\left(\bar{\gamma}^{\alpha}, \bar{\gamma}^{\beta}\right)$ a mixed strategy Epsilon Contracting Equilibrium iff $\left(\bar{\gamma}^{\alpha}(\epsilon), \bar{\gamma}^{\beta}(\epsilon)\right)$ is a mixed strategy $\epsilon$-equilibrium for each $\epsilon \in(0, \bar{\epsilon})$.

We denote the set of Epsilon Contracting Equilibria by $\Gamma$. Formally we have: $\Gamma=\{\bar{c} \mid \bar{c}$ is an ECE in the game among principals $\}$.

\section{Results}

Epsilon Contracting Equlibria are characterized in Propositions 1 and 2 below. The proofs for these propositions, as well as the intuition behind them, rely on a number of observations that are reported as Lemmas 1-3. Lemma 1 discusses a benchmark solution in which only one firm is active. ${ }^{9}$

Lemma 1. Suppose there is a single active firm. Then the optimal contract $c^{I}$ in this firm is such that $f_{1}=d_{1} ; f_{2}=d_{2} ; g_{11}=g_{12}=\phi\left(\theta_{1}, d_{1}\right) ; g_{21}=g_{22}=$ $\phi\left(\theta_{2}, d_{2}\right)+\phi\left(\theta_{1}, d_{1}\right)-\phi\left(\theta_{2}, d_{1}\right)$.

Proof: We first show that an optimal independent contract must induce the agent to play " $e\left(\theta_{1}\right)=d_{1} ; e\left(\theta_{2}\right)=d_{2}$ " in Stage 2 . Then we argue that the payout schedule specified in the lemma is the best payout schedule from the principal's point of view that triggers this behavior. First note that Assumption 2 implies that if the $\theta_{1}$-agent ${ }^{10}$ prefers to accept the contract at Stage 1 rather than to reject it, then the $\theta_{2}$-agent cannot prefer rejecting the contract rather than accepting. Next note that it cannot be optimal for the principal to let the agent who has observed $\theta_{1}$ reject the contract: If the $\theta_{2}$-agent rejects, too, the principal's ex ante payoff is 0 which is strictly less than $V_{P}\left(c^{I},-\right)$; if the $\theta_{2}$-agent accepts, the principal's ex ante payoff cannot exceed $\left(r_{21}+r_{22}\right) \phi\left(\theta_{2}, d_{2}\right)$; but (by Assumption 5) $\left(r_{21}+r_{22}\right) \phi\left(\theta_{2}, d_{2}\right)<$ $\left(r_{21}+r_{22}\right) \phi\left(\theta_{2}, d_{2}\right)+\phi\left(\theta_{1}, d_{1}\right)-\left(r_{21}+r_{22}\right) \phi\left(\theta_{2}, d_{1}\right)=V_{P}\left(c^{I},-\right)$. These observations reveal that an optimal independent contract must respect the participation constraints for both realizations of $\theta^{i}$. Next observe that an optimal independent contract cannot induce a pooling strategy, i.e., a strategy of the form " $e\left(\theta_{1}\right)=d_{k}$; $e\left(\theta_{2}\right)=d_{k}$ ": the principal's ex ante valuation of a contract that triggers such a behavior cannot exceed $\max \left\{\min \left[\phi\left(\theta_{1}, d_{1}\right), \phi\left(\theta_{2}, d_{1}\right)\right], \min \left[\phi\left(\theta_{1}, d_{2}\right), \phi\left(\theta_{2}, d_{2}\right)\right]\right\}=$ $\phi\left(\theta_{1}, d_{1}\right)$, which is strictly less than $V_{P}\left(c^{I},-\right)$. Thus, we are left with two possible strategies for the agent: " $e\left(\theta_{1}\right)=d_{1} ; e\left(\theta_{2}\right)=d_{2}$ " and " $e\left(\theta_{1}\right)=d_{2} ; e\left(\theta_{2}\right)=d_{1}$ ". Since the second of these strategies cannot be induced by any independent contract, the optimal independent contract must recommend " $f_{1}=d_{1} ; f_{2}=d_{2}$ ". The

\footnotetext{
${ }^{9}$ In the statement of Lemma 1 and in the rest of the paper the relation $\phi\left(\theta_{1}, d_{1}\right)>\left(r_{21}+\right.$ $\left.r_{22}\right) \phi\left(\theta_{2}, d_{1}\right)$ is supposed to hold. We refer to this as Assumption 5. It guarantees that the principal does not choose to ignore the agent who has observed $\theta_{1}$.

${ }^{10}$ We refer to an agent who observes $\theta_{h}(h=1,2)$ shortly as a $\theta_{h}$-agent.
} 
agent will obey this recommendation if and only if the associated payout schedule satisfies $\phi\left(\theta_{k}, d_{k}\right)-g_{k} \geq \phi\left(\theta_{k}, d_{l}\right)-g_{l}(k, l \in\{1,2\})$ and $\phi\left(\theta_{k}, d_{k}\right)-g_{k} \geq 0$ $(k \in\{1,2\})$, where $g_{k}=g_{k 1}=g_{k 2}$. Now it is straightforward to verify that the payout schedule characterized in the lemma is the best payout schedule from the principal's point of view that respects these relations.

We call the contract characterized in Lemma 1 the optimal independent contract and denote it by $c^{I}$. Note that if $P^{i}$ offers $c^{I}, A^{i}$ will accept the contract and play the recommended strategy. This follows from the relations $0=\phi\left(\theta_{1}, d_{1}\right)-$ $g_{11}>\phi\left(\theta_{1}, d_{2}\right)-g_{22}$ (by Assumption 3) and $\phi\left(\theta_{2}, d_{2}\right)-g_{22}=\phi\left(\theta_{2}, d_{1}\right)-g_{11}>0$ (by Assumption 2), and from WFL. ${ }^{11}$ From these relations we can also see that $A^{i}$ receives just his reservation utility when he observes $\theta_{1}^{i}$, while he gets a rent if $\theta^{i}=\theta_{2}^{i}$. The existence of this rent implies that $P^{i}$, s ex ante payoff under $c^{I}$ is lower than that in the first best solution where she would receive the whole surplus. She now gets $V_{P}\left(c^{I},-\right)=\phi\left(\theta_{1}, d_{1}\right)+\left(r_{21}+r_{22}\right)\left[\phi\left(\theta_{2}, d_{2}\right)-\phi\left(\theta_{2}, d_{1}\right)\right] .{ }^{12}$

Having identified $c^{I}$ as the optimal contract for a principal who deals with her agent in isolation, the first natural question is, of course, whether the strategy profile $\left(c^{I}, c^{I}\right)$ constitutes an ECE in the game among principals. The answer turns out to be no. The intuition is as follows: Under $c^{I}$ the agent in question is able to command a share of the surplus in the form of a rent. This implies that the principal would be strictly better off if she could observe the realization of $\theta^{i}$ along with her agent. Perfect observation is impossible. But even imperfect information is of some value. If one principal - say $P^{i}-$ commits to $c^{I}$, the Stage 2 behavior of $A^{i}$ provides such imperfect information. $A^{j}$ is, for example, more likely to observe $\theta_{2}^{j}$ if $A^{i}$ chooses $d_{2}$, rather than if he chooses $d_{1}$. This information is valuable to $P^{j}$ because she can use it to reduce $A^{j}$ 's informational rent. In other words, if $P^{i}$ signs $c^{I}, P^{j}$ has an incentive to choose a contract under which the payouts from $A^{j}$ depend not only on $d^{j}$ but also on $d^{i}$.

Lemma 2 lists some necessary conditions an optimal response to $c^{I}$ satisfies. $^{13}$ In the statement of this lemma and in what follows we write $U\left(g_{k l} \mid \theta_{h}\right)$ for $U\left[\phi\left(\theta_{h}, d_{k}\right)-g_{k l}\right]$. That is, $U\left(g_{k l} \mid \theta_{h}\right)$ is the utility level of an agent when his private information is $\theta_{h}$, his decision is $d_{k}$ and the decision of the other agent is $d_{l}$.

Lemma 2. Suppose $P^{i}$ signs $c^{I}$ and $\{i, j\}=\{\alpha, \beta\}$. Then the best response of $P^{j}$ satisfies the following conditions: ${ }^{14}$

$$
\begin{gathered}
f_{1}=d_{1} ; \quad f_{2}=d_{2} ; \\
R_{11}\left[U\left(g_{11} \mid \theta_{1}\right)-U\left(g_{21} \mid \theta_{1}\right)\right]+R_{12}\left[U\left(g_{12} \mid \theta_{1}\right)-U\left(g_{22} \mid \theta_{1}\right)\right]>0 ;
\end{gathered}
$$

\footnotetext{
${ }^{11}$ Here we utilize both $\mathrm{WFL}_{1}$ and $\mathrm{WFL}_{0} . \mathrm{WFL}_{1}$ is needed to guarantee that the $\theta_{2}$-agent plays $d_{2}$ in Stage 1 (although he is indifferent between playing $d_{2}$ and $d_{1}$ ), and $\mathrm{WFL}_{0}$ is needed to ensure that the $\theta_{1}$-agent accepts the contract (although he would get exactly the same monetary payoff by rejecting it).

12 Throughout the symbol $V_{P}^{i}\left(c^{i},-\right)$ is used if $c^{i}$ has a form that makes $P^{i}$ 's ex ante valuation of $c=\left(c^{\alpha}, c^{\beta}\right)$ independent of $c^{j}$ (no matter whether $A^{j}$ accepts $c^{j}$ or not).

13 Under the hypotheses of the model an optimal response to $c^{I}$ exists and this response is unique.

14 As also noted before, a best response is nothing but a 0-best response. The superscript $j$ is dropped since it is well-understood.
} 


$$
\begin{gathered}
R_{21}\left[U\left(g_{21} \mid \theta_{2}\right)-U\left(g_{11} \mid \theta_{2}\right)\right]+R_{22}\left[U\left(g_{22} \mid \theta_{2}\right)-U\left(g_{12} \mid \theta_{2}\right)\right]=0 ; \\
R_{21} U\left(g_{21} \mid \theta_{2}\right)+R_{22} U\left(g_{22} \mid \theta_{2}\right) \geq R_{11} U\left(g_{11} \mid \theta_{1}\right)+R_{12} U\left(g_{12} \mid \theta_{1}\right)=0 ; \\
U\left(g_{11} \mid \cdot\right)>U\left(g_{12} \mid \cdot\right) ; \quad U\left(g_{21} \mid \cdot\right)=U\left(g_{22} \mid \cdot\right) .
\end{gathered}
$$

Proof. Using arguments similar to those presented in the proof of Lemma 1, it can be shown that a best response to $c^{I}$ must contain the decision recommendation " $f_{1}=d_{1} ; f_{2}=d_{2}$ " ${ }^{15}$ For both types of the agent to accept the contract and to obey this recommendation, the accompanying payout function must satisfy:

$$
\begin{array}{ll}
R_{k 1}\left[U\left(g_{k 1} \mid \theta_{k}\right)-U\left(g_{l 1} \mid \theta_{k}\right)\right]+R_{k 2}\left[U\left(g_{k 2} \mid \theta_{k}\right)\right. & \\
\left.\quad-U\left(g_{l 2} \mid \theta_{k}\right)\right] \geq 0 \quad(\{k, l\}=\{1,2\}) ; & \left(S S_{k}\right) \\
R_{k 1} U\left(g_{k 1} \mid \theta_{k}\right)+R_{k 2} U\left(g_{k 2} \mid \theta_{k}\right) \geq 0 \quad(k \in\{1,2\}) . & \left(I R_{k}\right)
\end{array}
$$

Among the payout schedules which satisfy these constraints, the one that is optimal from the principal's point of view chooses $\left(g_{11}, g_{12}, g_{21}, g_{22}\right)$ so as to maximize $\sum_{k=1}^{2} \sum_{l=1}^{2} r_{k l} g_{k l}$ subject to $S S_{1}, S S_{2}, I R_{1}$ and $I R_{2}$. Denote this program by $W$. In the search for a solution to $W$, we first consider a relaxed program $R P$ in which $S S_{1}$ is not included. Later we will verify that $S S_{1}$ is, in fact, satisfied by the solution to $R P$. First, observe that at a solution to $R P, I R_{1}$ and $S S_{2}$ are both binding: if $I R_{1}$ were slack, it would be possible to raise $g_{11}$ slightly which does not violate $I R_{1}$, relaxes $S S_{2}$ and increases the objective. If $S S_{2}$ were slack, then it would be possible for the principal to move to another payout schedule $\left(\tilde{g}_{11}, \tilde{g}_{12}, \tilde{g}_{21}, \tilde{g}_{22}\right)$ on the line segment joining $\left(g_{11}, g_{12}, g_{21}, g_{22}\right)$ to the first best solution $\left(\hat{g}_{11}, \hat{g}_{12}, \hat{g}_{21}, \hat{g}_{22}\right)$ with $\hat{g}_{11}=\hat{g}_{12}=\phi\left(\theta_{1}, d_{1}\right) ; \hat{g}_{21}=\hat{g}_{22}=\phi\left(\theta_{2}, d_{2}\right)$ which still satisfies the constraints and yields a higher ex ante payoff to the principal than does the current payout schedule. Next, observe that with $I R_{1}$ and $S S_{2}$ binding, $R P$ is a strictly concave program (the relevant assumptions here are (2), (3), (4) and NIARA) so that a unique solution exists. Finally, observe that at the solution we have $g_{21}=g_{22}$ : if $g_{21} \neq g_{22}$, then an improvement could be obtained by replacing $g_{21}$ and $g_{22}$ by $\bar{g}_{2}$, where $\bar{g}_{2}$ is such that $U\left(\bar{g}_{2} \mid \theta_{2}\right)=$ $R_{21} U\left(g_{21} \mid \theta_{2}\right)+R_{22} U\left(g_{22} \mid \theta_{2}\right)$; all the constraints would continue to be met and since $U(\cdot)$ is strictly concave, $\bar{g}_{2}>R_{21} g_{21}+R_{22} g_{22}$. It remains to be shown that $g_{12}>g_{11}$. To prove this, we analyze the FOC s associated with $R P$. Let $\mu_{2}>0$ denote the multiplier for $S S_{2}$, and $\lambda_{1}>0$ that for $I R_{1}$. The FOC s for $g_{11}$ and $g_{12}$ are: $r_{11}+\mu_{2} R_{21} U^{\prime}\left(g_{11} \mid \theta_{2}\right)-\lambda_{1} R_{11} U^{\prime}\left(g_{11} \mid \theta_{1}\right)=0$ and

\footnotetext{
15 There is one major difference in the argumentation: In the proof of Lemma 1, we conclude that an optimal independent contract cannot induce a pooling strategy of the agent by comparing the principal's ex ante valuation of a contract inducing such a behavior with her valuation of $c^{I}$. Here (and in the proof of Lemma 3) a comparison with $c^{I}$ leads to the desired conclusion only for " $e\left(\theta_{1}\right)=e\left(\theta_{2}\right)=d_{2}$ ". For " $e\left(\theta_{1}\right)=e\left(\theta_{2}\right)=d_{1}$ " the following contract, denoted by $\hat{c}$, plays the role of $c^{I}$ in Lemma 1: $\hat{c}=\left(\hat{f}_{1}, \hat{f}_{2}, \hat{g}_{11}, \hat{g}_{12}, \hat{g}_{21}, \hat{g}_{22}\right)$ with $\hat{f}_{1}=d_{1}, \hat{f}_{2}=d_{2}, \hat{g}_{11}=g_{11}, \hat{g}_{12}=g_{12}$, $\hat{g}_{21}=g_{11}+\phi\left(\theta_{2}, d_{2}\right)-\phi\left(\theta_{2}, d_{1}\right)-\gamma, \hat{g}_{22}=g_{12}+\phi\left(\theta_{2}, d_{2}\right)-\phi\left(\theta_{2}, d_{1}\right)-\gamma$, where $0<\gamma<$ $\phi\left(\theta_{2}, d_{2}\right)-\phi\left(\theta_{2}, d_{1}\right)$ and where $g_{11}$ (or $g_{12}$, respectively) is the transfer specified in the original (pooling) contract for the situation in which the agent under consideration chooses $d_{1}$ and the second agent chooses $d_{1}$ (or $d_{2}$, respectively).
} 
$r_{12}+\mu_{2} R_{22} U^{\prime}\left(g_{12} \mid \theta_{2}\right)-\lambda_{1} R_{12} U^{\prime}\left(g_{12} \mid \theta_{1}\right)=0$. Solving for $\lambda_{1}$ and subtracting the second equation from the first yields:

$$
\begin{aligned}
& \left(r_{21}+r_{22}\right)\left[\frac{1}{U^{\prime}\left(g_{11} \mid \theta_{1}\right)}-\frac{1}{U^{\prime}\left(g_{12} \mid \theta_{1}\right)}\right] \\
& =\mu_{2}\left[\frac{r_{22}}{r_{12}} \frac{U^{\prime}\left(g_{12} \mid \theta_{2}\right)}{U^{\prime}\left(g_{12} \mid \theta_{1}\right)}-\frac{r_{21}}{r_{11}} \frac{U^{\prime}\left(g_{11} \mid \theta_{2}\right)}{U^{\prime}\left(g_{11} \mid \theta_{1}\right)}\right] .
\end{aligned}
$$

Consider first the $R H S$ of $(\dagger)$. Suppose $g_{11} \geq g_{12}$. Assumption 2 implies that $U\left(g_{k l} \mid \theta_{2}\right)>U\left(g_{k l} \mid \theta_{1}\right)$ for each $g_{k l}$. Therefore, by NIARA, $a \equiv U^{\prime}\left(g_{12} \mid \theta_{2}\right) /$ $U^{\prime}\left(g_{12} \mid \theta_{1}\right) \geq U^{\prime}\left(g_{11} \mid \theta_{2}\right) / U^{\prime}\left(g_{11} \mid \theta_{1}\right) \equiv b>0 .{ }^{16}$ Moreover, by Assumption 4, $c \equiv r_{22} / r_{12}>r_{21} / r_{11} \equiv d>0$, so that $a c>b d$. Thus, the RHS of $(\dagger)$ is positive. Now consider the $L H S$. From $g_{11} \geq g_{12}$ and the concavity of $U(\cdot)$, $U^{\prime}\left(g_{11} \mid \theta_{1}\right) \geq U^{\prime}\left(g_{12} \mid \theta_{1}\right)$. But then the LHS of $(\dagger)$ is nonpositive. This contradiction proves that $g_{12}>g_{11}$. It remains only to verify that at a solution to $R P$ the missing $S S_{1}$-constraint is satisfied. To show this, let $c^{R P}$ denote the contract $\left(f_{1}=d_{1} ; f_{2}=d_{2} ; g_{11}, g_{12}, g_{21}, g_{22}\right)$, where $\left(g_{11}, g_{12}, g_{21}, g_{22}\right)$ is the solution to $R P$. Also let $\dot{c}=\left(\dot{g}_{11}, \dot{g}_{12}, \dot{g}_{21}, \dot{g}_{22}\right)$ be a vector in which $\dot{g}_{11}=\dot{g}_{12}=\hat{g}_{11}$ and $\dot{g}_{21}=\dot{g}_{22}=\hat{g}_{22}$, where $\hat{g}_{11}$ and $\hat{g}_{22}$ are as defined in Lemma 1. First note that since $\dot{c}$ is feasible as a solution to $R P$ but not optimal, we have that $V_{P}\left(c^{R P}, c^{I}\right)>V_{P}\left(c^{I},-\right)$. Furthermore, from the arguments in the proof of Lemma $1, V_{P}\left(c^{I},-\right)>V_{P}(\bar{c},-)$ for all $\bar{c}$, where $\bar{c}$ denotes an independent pooling contract in which the agent is instructed to play $d_{2}$ for all realizations of $\theta^{i}$ and in which $\bar{g}_{1}$ and $\bar{g}_{2}$ (the transfers from $A$ to $P$ for $d_{1}$ and $d_{2}$ ) satisfy: $\bar{g}_{1} \geq \bar{g}_{2}+\phi\left(\theta_{1}, d_{1}\right)-\phi\left(\theta_{1}, d_{2}\right)$. Our aim is to show that, for each solution to $R P$ in which $S S_{1}$ is violated, one has $V_{P}\left(c^{R P}, c^{I}\right)<V_{P}(\bar{c},-)$ for some $\bar{c}$. To see this, replace $c^{R P}$ by a $\bar{c}$ in which $\bar{g}_{2}=g_{21}$ and $\bar{g}_{1}=\bar{g}_{2}+\phi\left(\theta_{1}, d_{1}\right)-\phi\left(\theta_{1}, d_{2}\right)+\epsilon$ for some $\epsilon>0$. Admissibility of $c^{R P}$ as a solution to $R P$, together with the supposition that $S S_{1}$ is violated, guarantees that the agent gets his reservation utility for each realization of the random variable under $\bar{c}$, so that the replacement is feasible. Utilizing the facts that $S S_{2}$ is binding, $g_{11} \neq g_{12}, g_{21}=g_{22}$, and $U$ is strictly concave, we get that $\phi\left(\theta_{2}, d_{2}\right)-g_{21}<R_{21}\left[\phi\left(\theta_{2}, d_{1}\right)-g_{11}\right]+R_{22}\left[\phi\left(\theta_{2}, d_{1}\right)-g_{12}\right]$. Therefore, $g_{21}+\phi\left(\theta_{2}, d_{1}\right)-\phi\left(\theta_{2}, d_{2}\right)>R_{21} g_{11}+R_{22} g_{12}>R_{11} g_{11}+R_{12} g_{12}$, where the last inequality follows from $R_{11}>R_{21}$ and $g_{12}>g_{11}$. By Assumption 3, $\phi\left(\theta_{2}, d_{1}\right)-\phi\left(\theta_{2}, d_{2}\right)<0$. Combined with the definition of $\bar{g}_{2}$, this gives $\bar{g}_{2}=$ $g_{21}>R_{11} g_{11}+R_{12} g_{12}$, so that the change in the objective obtained by the replacement is positive. This contradicts the optimality of the $S S_{1}$-violating solution to $R P$.

We call the best response to $c^{I}$ the optimal weak comparative contract and denote it by $c^{W}$. If $P^{i}$ commits to $c^{I}$ and $P^{j}$ offers $c^{W}$, then $A^{j}$ will accept the

\footnotetext{
16 To see this, define $\psi(x) \equiv\left[U^{\prime}(x+\Delta) / U^{\prime}(x)\right]$, where $\Delta>0$. Then $\psi^{\prime}(x) \geq 0 \Longleftrightarrow$ $U^{\prime \prime}(x+\Delta) / U^{\prime}(x+\Delta) \geq U^{\prime \prime}(x) / U^{\prime}(x) \Longleftrightarrow U^{\prime \prime}(x) / U^{\prime}(x)$ is a non-decreasing function $\Longleftrightarrow$ $-\left[U^{\prime \prime}(x) / U^{\prime}(x)\right]$ is a non-increasing function.
} 
contract and play the recommended strategy. This follows from the relations 6,7 and 8 and from WFL. ${ }^{17}$ Relations 6 and 7 reflect the requirement that, conditional on his private information and the belief that the other agent obeys the decision recommendation of $c^{I}$, the agent under $c^{W}$ does not prefer to adopt a strategy other than that designated for him; i.e., given $e^{i}(\cdot)=\left(e^{i}\left(\theta_{1}^{i}\right)=d_{1} ; e^{i}\left(\theta_{2}^{i}\right)=d_{2}\right)$, playing $e^{j}=f^{j}$ is a best Bayes-Nash-response for $A^{j}$. Equation 7 tells us that the binding incentive problem is to prevent the $\theta_{2}$-agent from mimicking the $\theta_{1}$-agent. From condition 9 we can see how this incentive problem is mitigated under $c^{W}$. This contract offers the agent who chooses $d_{1}$ a relatively low payoff if the other agent plays $d_{2}$ and a higher payoff if the other agent chooses $d_{1}$; $U\left(g_{11} \mid \cdot\right)>U\left(g_{12} \mid \cdot\right)$ helps with incentives because if $A^{j}$ observes $\theta_{1}^{j}$ and plays $d_{1}$, then he knows that $\theta^{i}$ is relatively unlikely to be $\theta_{2}^{i}$ (and thus, $A^{i}$ is relatively unlikely to choose $d_{2}$ ), and so he is unlikely to suffer the "penalty" $g_{12}$; but if $A^{j}$ observes $\theta_{2}^{j}$ and behaves as if he had observed $\theta_{1}^{j}$ the penalty $g_{12}$ is more likely. This "screening by expectations" easiers the binding incentive problem and thereby reduces the agent's information rent. As a result, given $c^{i}=c^{I}, P^{j}$ 's ex ante payoff under $c^{W}$ is higher than that under $c^{I}: V_{P}^{j}\left(c^{W}, c^{I}\right)>V_{P}^{j}\left(c^{I},-\right)$.

In the situation just considered the behavior of one agent produces an informational externality which enables the principal of the other hierarchy to take advantage of the correlation between the random variables. Clearly, both principals would prefer to sign $c^{W}$ provided that the players in the other firm commit to $c^{I}$. A natural next question therefore is whether the contract pair $\left(c^{W}, c^{I}\right)$ (or its mirror image) constitutes an ECE in the game among principals.

Again the answer is no, and the intuition seems to be clear. Now $c^{W}$ induces the agent who has accepted it to play the perfectly revealing strategy " $e\left(\theta_{1}\right)=d_{1}$; $e\left(\theta_{2}\right)=d_{2}$ " as a Bayes-Nash response to the same strategy chosen by the agent under $c^{I}$. Since $c^{W}$ is the best response to $c^{I}$ and since $c^{I}$ and $c^{W}$ induce the same behavior in the agents' game, one might think that $c^{W}$ should be a best response to $c^{W}$, too.

However, the matter is somewhat more complicated. If both principals commit to $c^{W}$, the constraints in the resulting game ensure that playing the recommended separating strategies forms a $B E$ in the agents' game. However, there is also another $\mathrm{BE}$ of this game in which $e^{i}(\cdot)=d_{1}$ for each $\theta^{i} \in \Theta^{i}$ and each $i \in\{\alpha, \beta\}$, that is, in which each agent always acts as if he had observed $\theta_{1}^{i}$. To see that these strategies form an equilibrium, first remember that $c^{W}$ has $g_{12}>g_{11}$ and $g_{21}=g_{22}$. Hence, using $6, U\left(g_{11} \mid \theta_{1}\right)>R_{11} U\left(g_{11} \mid \theta_{1}\right)+R_{12} U\left(g_{12} \mid \theta_{1}\right)>$ $U\left(g_{21} \mid \theta_{1}\right)$. Similarly, $U\left(g_{11} \mid \theta_{2}\right)>R_{21} U\left(g_{11} \mid \theta_{2}\right)+R_{22} U\left(g_{12} \mid \theta_{2}\right)=U\left(g_{21} \mid \theta_{2}\right)$, where the equality follows from Eqs. 7 and 9. From these relations we can also see that the utility of each agent at each $\theta^{i}$-realization goes strictly up upon moving from the recommended to the "undesired" BE. Thus, given the assumption of Payoff Dominance, the agents will focus on the latter. Since the principals could

\footnotetext{
${ }^{17}$ Under the contract combination $\left(c^{I}, c^{W}\right) \mathrm{WFL}_{1}^{\alpha}$ is used to eliminate the $\mathrm{BE}$ " $e^{\alpha}(\cdot)=e^{\beta}(\cdot)=$ $\left(e\left(\theta_{1}\right)=d_{1} ; e\left(\theta_{2}\right)=d_{1}\right)$ " and $\mathrm{WFL}_{1}^{\beta}$ to eliminate the $\mathrm{BE}$ " $e^{\alpha}(\cdot)=\left(e\left(\theta_{1}\right)=d_{1} ; e\left(\theta_{2}\right)=d_{2}\right) ; e^{\beta}(\cdot)=$ $\left(e\left(\theta_{1}\right)=d_{1} ; e\left(\theta_{2}\right)=d_{1}\right) . "$
} 
improve their position by raising $g_{11}$ without causing any quittings, $\left(c^{W}, c^{W}\right)$ cannot form an ECE. ${ }^{18}$

The question therefore remains: What is $P^{i}$, s (epsilon) best response to $c^{W}$ ? Lemma 3 deals with this question:

Lemma 3. Suppose $P^{j}$ signs $c^{W}$. For any Epsilon Best Response $\bar{c}^{i}:[0, \bar{\epsilon}) \rightarrow C^{i}$ (where $\bar{\epsilon}>0$ ) of $P^{i}$ to $c^{W}, \bar{c}^{i}(0)=\left(f_{1}, f_{2}, g_{11}, g_{12}, g_{21}, g_{22}\right)$ satisfies conditions 5 , 6 and 8 of Lemma 2 and

$$
U\left(g_{21} \mid \theta_{2}\right)=U\left(g_{11} \mid \theta_{2}\right)>U\left(g_{12} \mid \theta_{2}\right)=U\left(g_{22} \mid \theta_{2}\right) .
$$

Moreover, there exists an Epsilon Best Response $\tilde{c}^{i}:[0, \tilde{\epsilon}) \rightarrow C^{i}$ (with $\tilde{\epsilon}>0$ ) of $P^{i}$ to $c^{W}$ of the form

$$
\tilde{c}^{i}(\epsilon)=\left(\tilde{f}_{1}, \tilde{f}_{2}, \tilde{g}_{11}, \tilde{g}_{12}, \tilde{g}_{21}-\epsilon, \tilde{g}_{22}-\epsilon\right)
$$

for each $\epsilon \in[0, \tilde{\epsilon}) .(\{i, j\}=\{\alpha, \beta\})$

Proof. Take any Epsilon Best Response (EBR) $\bar{c}^{i}:[0, \bar{\epsilon}) \rightarrow C^{i}$ of $P^{i}$ to $c^{W}$, where $\bar{c}^{i}(\epsilon)=\left(\bar{f}_{1}, \bar{f}_{2}, \bar{g}_{11}(\epsilon), \bar{g}_{12}(\epsilon), \bar{g}_{21}(\epsilon), \bar{g}_{22}(\epsilon)\right)$ for all $\epsilon \in[0, \bar{\epsilon})$. It follows from arguments similar to those in the proof of Lemma 1 and in Footnote 15 that the decision recommendation in $\bar{c}^{i}$ is " $\bar{f}_{1}=d_{1} ; \bar{f}_{2}=d_{2}$ ", whenever $\epsilon \in(0, \bar{\epsilon}] \cap\left(0, \frac{1}{2}\left(r_{21}+r_{22}\right)\left(\phi\left(\theta_{2}, d_{2}\right)-\phi\left(\theta_{2}, d_{1}\right)\right)\right.$. ${ }^{19}$ Since $\bar{f}_{1}$ and $\bar{f}_{2}$ are constant by definition of an EBR, it is no loss of generality to assume that $\bar{\epsilon} \in\left(0, \frac{1}{2}\left(r_{21}+r_{22}\right)\left(\phi\left(\theta_{2}, d_{2}\right)\right)-\phi\left(\theta_{2}, d_{1}\right)\right)$. But then $\left(\bar{g}_{11}(\epsilon), \bar{g}_{12}(\epsilon), \bar{g}_{21}(\epsilon), \bar{g}_{22}(\epsilon)\right)$ satisfies $S S_{1}, S S_{2}, I R_{1}$, and $I R_{2}$ (as defined in the proof of Lemma 2) for each $\epsilon \in(0, \bar{\epsilon})$. So, $\left(\bar{g}_{11}(0), \bar{g}_{12}(0), \bar{g}_{21}(0), \bar{g}_{22}(0)\right)$ satisfies the same conditions as well by continuity of $\bar{g}_{k l}(k, l \in\{1,2\})$ and $U$. (To simplify notation, we will denote $\bar{g}_{k l}(0)$ just by $\bar{g}_{k l}(k, l \in\{1,2\})$ henceforth. $)$

Now note that, for $A^{i}$ who is offered $\bar{c}^{i}(\epsilon)$ to accept this contract and follow its decision recommendation under $\left(\bar{c}^{i}(\epsilon), c^{W}\right)$, one must have in addition to $S S_{1}, S S_{2}, I R_{1}, I R_{2}$ that either (a) playing " $e^{t}\left(\theta_{1}^{t}\right)=e^{t}\left(\theta_{2}^{t}\right)=d_{1}$ " for each $t \in\{\alpha, \beta\}$ is not a $\mathrm{BE}$ in the agents' game or (b) it forms a $\mathrm{BE}$ without dominating the recommended equilibrium in view of our assumption of Payoff Dominance. Remembering that an agent under $c^{W}$ will always respond to " $e\left(\theta_{1}\right)=e\left(\theta_{2}\right)=d_{1}$ " by the same pooling strategy, (a) is seen to be equivalent to the disjunction of $\left(a_{1}\right)$ and $\left(a_{2}\right)$, where $\left(a_{1}\right)$ is $U\left(\bar{g}_{21}(\epsilon) \mid \theta_{1}\right)>U\left(\bar{g}_{11}(\epsilon) \mid \theta_{1}\right)$, and $\left(a_{2}\right)$ is $U\left(\bar{g}_{21}(\epsilon) \mid \theta_{2}\right)>U\left(\bar{g}_{11}(\epsilon) \mid \theta_{2}\right)$. However, since $\left(a_{1}\right)$ is strictly more demanding than $\left(a_{2}\right)$ by Assumption 3, we conclude that (a) is actually equivalent to $\left(a_{2}\right)$.

We now want to show that there is some $\bar{\epsilon}^{\prime} \in(0, \bar{\epsilon}]$ such that $\left(a_{2}\right)$ holds for all $\epsilon \in\left(0, \bar{\epsilon}^{\prime}\right)$. Suppose not. Then, for each $n \in \mathbf{N}$ (where $\mathbf{N}$ stands for the set of

\footnotetext{
18 Strictly speaking, our reasoning here indicates that $\left(c^{W}, c^{W}\right)$ does not form a CE. However, it is easy to conclude that $\left(c^{W}, c^{W}\right)$ actually is not an ECE either, for one has to consider in an epsilon equilibrium what happens when $\epsilon>0$ is smaller than the difference of a principal's payoffs induced by the recommended and the undesirable agents' BE, respectively, as well.

19 Since we now start with an EBR the limits for $\gamma$ in the contract $\hat{c}$ defined in Footnote 15 have to be modified. Restricting $\gamma$ to the same interval as $\epsilon$ will do the job.
} 
natural numbers), there is some $\epsilon_{n} \in\left(0, \frac{1}{n}\right) \cap(0, \bar{\epsilon})$ for which $\left(a_{2}\right)$ does not hold. But then (b) holds for each such $n$. Moreover, $\lim _{n \rightarrow \infty} \bar{c}^{i}\left(\epsilon_{n}\right)=\bar{c}^{i}(0)$ since clearly $\epsilon_{n} \rightarrow 0 .^{20}$ Also note that

$$
V_{P}^{i}\left(c^{I},-\right)=V_{P}^{i}\left(c^{I}, c^{W}\right) \leq V_{P}^{i}\left(\bar{c}^{i}\left(\epsilon_{n}\right), c^{W}\right)+\epsilon_{n}
$$

for each $n \in \mathbf{N}$, since $\bar{c}^{i}\left(\epsilon_{n}\right)$ is an $\epsilon_{n}$-best response to $c^{W}$ by definition of an EBR.

Now consider the program of maximizing $\sum_{i=1}^{2} \sum_{j=1}^{2} r_{i j} g_{i j}$ subject to $S S_{1}, S S_{2}, I R_{1}$, $I R_{2}$ and (b). Proceeding similarly as in the proof of Lemma 2, it can be checked that $c^{I}$ is a solution to this program. Now since $\left(\bar{g}_{11}\left(\epsilon_{n}\right), \bar{g}_{12}\left(\epsilon_{n}\right), \bar{g}_{21}\left(\epsilon_{n}\right), \bar{g}_{22}\left(\epsilon_{n}\right)\right)$ is a feasible payoff schedule for the above program and $\left(\bar{c}^{i}\left(\epsilon_{n}\right), c^{W}\right)$ leads to the recommended separating $\mathrm{BE}$ in the agents' game for all $n \in \mathbf{N}$, we have

$$
V_{P}^{i}\left(\bar{c}^{i}\left(\epsilon_{n}\right), c^{W}\right) \leq V_{P}^{i}\left(c^{I}, c^{W}\right)
$$

for each such $n$. So, combining this with the inequality obtained above, we have

$$
V_{P}^{i}\left(c^{I}, c^{W}\right)-\epsilon_{n} \leq V_{P}^{i}\left(\bar{c}^{i}\left(\epsilon_{n}\right), c^{W}\right) \leq V_{P}^{i}\left(c^{I}, c^{W}\right)
$$

for all $n \in \mathbf{N}$ which, in turn, implies that

$$
\lim _{n \rightarrow \infty} V_{P}^{i}\left(\bar{c}^{i}\left(\epsilon_{n}\right), c^{W}\right)=V_{P}^{i}\left(c^{I}, c^{W}\right)=V_{P}^{i}\left(c^{I},-\right) .
$$

Now let $\left(a_{2}^{\prime}\right)$ stand for the condition $U\left(g_{12} \mid \theta_{2}\right) \geq U\left(g_{11} \mid \theta_{2}\right)$ obtained from $\left(a_{2}\right)$ by replacing strict inequality by a weak one. Define Program $S$ as follows: Maximize $\sum_{j=1}^{2} \sum_{i=1}^{2} r_{i j} g_{i j}$ subject to $S S_{1}, S S_{2}, I R_{1}, I R_{2}$ and $\left(a_{2}^{\prime}\right)$. Let $\left(\tilde{g}_{11}, \tilde{g}_{12}, \tilde{g}_{21}, \tilde{g}_{22}\right)$ be a solution to Program $S$. Moreover, define $\tilde{c}^{S}:[0, \bar{\epsilon}) \rightarrow C^{i}$ by $\tilde{c}^{S}(\epsilon)=$ $\left(\tilde{f}_{1}, \tilde{f}_{2}, \tilde{g}_{11}, \tilde{g}_{12}, \tilde{g}_{21}-\epsilon, \tilde{g}_{22}-\epsilon\right)$ for each $\epsilon \in[0, \bar{\epsilon})$, where $\tilde{f}_{1}=d_{1}, \tilde{f}_{2}=d_{2}$. Notice that " $e\left(\theta_{1}\right)=d_{1} ; e\left(\theta_{2}\right)=d_{2}$ " is the unique dominant strategy for an agent under $\tilde{c}^{S}(\epsilon)$ whenever $\epsilon \in(0, \bar{\epsilon})$, whereas, for an agent under $c^{W}$, along with the pooling strategy " $e\left(\theta_{1}\right)=e\left(\theta_{2}\right)=d_{2}$ ", it is a best response to the same behavior of the other agent. Now WFL eliminates the BE where $e^{i}\left(\theta_{1}^{i}\right)=d_{1}, e^{i}\left(\theta_{2}^{i}\right)=d_{2}$ and $e^{j}\left(\theta_{1}^{j}\right)=e^{j}\left(\theta_{2}^{j}\right)=d_{1}$. Thus, for each $\epsilon \in(0, \bar{\epsilon}),\left(\tilde{c}^{S}(\epsilon), c^{W}\right)$ induces the recommended separating $\mathrm{BE}$ in the agents' game.

Next notice that the payoff schedule of $c^{I}$ is feasible, but not optimal for Program $S$, so that $V_{P}^{i}\left(\tilde{c}^{S}(0), c^{I}\right)>V_{P}^{i}\left(c^{I},-\right)$ since $\tilde{c}^{S}(0)$ is a solution to Program $S$. Moreover,

$$
\lim _{\epsilon \rightarrow 0} V_{P}^{i}\left(\tilde{c}^{S}(\epsilon), c^{W}\right)=\lim _{\epsilon \rightarrow 0} V_{P}^{i}\left(\tilde{c}^{S}(\epsilon), c^{I}\right)=V_{P}^{i}\left(\tilde{c}^{S}(0), c^{I}\right)
$$

\footnotetext{
${ }^{20}$ Note that to talk of the limit of a sequence of contracts each of which is a member of $D^{i} \times D^{i} \times \mathbf{R}^{4}$, one needs to introduce a metric structure on $D^{i}$ as well. We assume that the set $\left\{d_{1}, d_{2}\right\}$ is endowed with the discrete metric whenever such a structure is formally needed.
} 
since $\left(\tilde{c}^{S}(\epsilon), c^{W}\right)$ induces the same BE for all $\epsilon \in(0, \bar{\epsilon})$ which coincides with the BE induced by $\left(\tilde{c}^{S}(\epsilon), c^{I}\right)$ for all $\epsilon \in[0, \bar{\epsilon}) .{ }^{21}$ Thus, there exists some $\bar{\epsilon}^{\prime \prime} \in(0, \bar{\epsilon})$ such that $V_{P}^{i}\left(\tilde{c}^{S}(\epsilon), c^{W}\right)>V_{P}^{i}\left(c^{I},-\right)$ for all $\epsilon \in\left(0, \bar{\epsilon}^{\prime \prime}\right)$. Choose and fix some $\epsilon_{0} \in\left(0, \bar{\epsilon}^{\prime \prime}\right)$ and set $\delta=\frac{1}{2}\left(V_{P}^{i}\left(\tilde{c}^{S}\left(\epsilon_{0}\right), c^{W}\right)-V_{P}^{i}\left(c^{I},-\right)\right)>0$. So, $V_{P}^{i}\left(c^{I},-\right)+\delta<$ $V_{P}^{i}\left(\tilde{c}^{S}\left(\epsilon_{0}\right), c^{W}\right)$. Moreover, because of $(*)$, there is some sufficiently large $n$ so that $V_{P}^{i}\left(\bar{c}^{i}\left(\epsilon_{n}\right), c^{W}\right)-V_{P}^{i}\left(c^{I},-\right)<\frac{\delta}{2}$ and $\epsilon_{n}<\frac{\delta}{2}$. But then $V_{P}^{i}\left(\bar{c}^{i}\left(\epsilon_{n}\right), c^{W}\right)-$ $V_{P}^{i}\left(c^{I},-\right)+\epsilon_{n}<\delta<V_{P}^{i}\left(\tilde{c}^{S}\left(\epsilon_{0}\right), c^{W}\right)-V_{P}^{i}\left(c^{I},-\right)$, i.e. $V_{P}^{i}\left(\bar{c}^{i}\left(\epsilon_{n}\right), c^{W}\right)+\epsilon_{n}<$ $V_{P}^{i}\left(\tilde{c}^{S}\left(\epsilon_{0}\right), c^{W}\right)$, contradicting that $\bar{c}^{i}\left(\epsilon_{n}\right)$ is an $\epsilon_{n}$-best response to $c^{W}$. Thus, there is some $\bar{\epsilon}^{\prime} \in(0, \bar{\epsilon}]$ such that $\left(a_{2}\right)$ holds under $\left(\bar{c}^{i}(\epsilon), c^{W}\right)$ for all $\epsilon \in\left(0, \bar{\epsilon}^{\prime}\right)$. Thus, for all such $\epsilon$,

$$
U\left(\bar{g}_{21}(\epsilon) \mid \theta_{2}\right)>U\left(\bar{g}_{11}(\epsilon) \mid \theta_{2}\right),
$$

implying that $\left(a_{2}^{\prime}\right)$, i.e., $U\left(\bar{g}_{21} \mid \theta_{2}\right) \geq U\left(\bar{g}_{11} \mid \theta_{2}\right)$, holds by continuity of $\bar{g}_{21}(\epsilon)$, $\bar{g}_{11}(\epsilon)$ and $U$.

In summary, $\left(\bar{g}_{11}, \bar{g}_{12}, \bar{g}_{21}, \bar{g}_{22}\right)$ satisfies $S S_{1}, S S_{2}, I R_{1}, I R_{2}$ and $\left(a_{2}^{\prime}\right)$, i.e., it is feasible for Program $S$. Now since $\left(\bar{c}^{i}(\epsilon), c^{W}\right)$ induces the same separating BE in the agents' game for all $\epsilon \in\left(0, \bar{\epsilon}^{\prime}\right)$ which coincides with the BE induced under $\left(\bar{c}^{i}(0), c^{I}\right)$, we also have that

$$
\lim _{\epsilon \rightarrow 0} V_{P}^{i}\left(\bar{c}^{i}(\epsilon), c^{W}\right)=V_{P}^{i}\left(\bar{c}^{i}(0), c^{I}\right) .
$$

Finally, the above limit is equal to $\operatorname{Sup}_{c^{i} \in C^{i}} V_{P}^{i}\left(c^{i}, c^{W}\right)$ since $\bar{c}^{i}$ is an EBR to $c^{W}$. Since also $V_{P}^{i}\left(\bar{c}^{i}(0), c^{I}\right)=\sum_{i=1}^{2} \sum_{j=1}^{2} r_{i j} \bar{g}_{i j}$, we conclude that $\left(\bar{g}_{11}, \bar{g}_{12}, \bar{g}_{21}, \bar{g}_{22}\right)$ is a solution to Program $S$.

To show that a solution to Program $S$ satisfies the conditions specified in this lemma, we first consider a relaxed version $R V$ of this program in which $S S_{1}$ is not included. We then verify that $S S_{1}$ is also satisfied by a solution to $R V$. Proceeding similarly as in the proof of Lemma 2 , it is seen that $I R_{1}$ and $S S_{2}$ are binding. Note that, if we also exclude $\left(a_{2}^{\prime}\right), R V$ reduces to Program $R P$ considered in the proof of Lemma 2 a solution of which satisfies $U\left(g_{11} \mid \theta_{2}\right)>U\left(g_{12} \mid \theta_{2}\right)$ and $U\left(g_{21} \mid \theta_{2}\right)=U\left(g_{22} \mid \theta_{2}\right)$. These conjoined with $S S_{2}$, however, imply that such a solution violates $\left(a_{2}^{\prime}\right)$, leading to the conclusion that $\left(a_{2}^{\prime}\right)$ is also binding in $R V$.

From $S S_{2}$ and $\left(a_{2}^{\prime}\right)$ binding we get $U\left(g_{21} \mid \theta_{2}\right)=U\left(g_{11} \mid \theta_{2}\right)$ and $U\left(g_{22} \mid \theta_{2}\right)=$ $U\left(g_{12} \mid \theta_{2}\right)$ for a solution $\left(g_{11}, g_{12}, g_{21}, g_{22}\right)$ to $R V$. To prove that $U\left(g_{21} \mid \cdot\right)>$ $U\left(g_{22} \mid \cdot\right)$, we use the FOCs associated with $R V$. Denoting the multiplier for $\left(a_{2}\right)$ by $a$, for $S S_{2}$ by $\mu_{2}$ and for $I R_{2}$ by $\lambda_{2}$, we get $r_{21}-\left(\mu_{2} R_{21}+a\right) U^{\prime}\left(g_{21} \mid \theta_{2}\right)-$ $\lambda_{2} R_{21} U^{\prime}\left(g_{21} \mid \theta_{2}\right)=0$ and $r_{22}-\mu_{2} R_{22} U^{\prime}\left(g_{22} \mid \theta_{2}\right)-\lambda_{2} R_{22} U^{\prime}\left(g_{22} \mid \theta_{2}\right)=0$. Solving for $\lambda_{2}$ and subtracting the second equation from the first yields $1 / U^{\prime}\left(g_{21} \mid \theta_{2}\right)-$

\footnotetext{
${ }^{21}$ Here it is important to notice that $V_{P}^{i}$ is not continuous at each point of its domain $C^{i} \times C^{j}$, one such particular point of discontinuity being the contract pair $\left(\tilde{c}^{S}(0), c^{W}\right)$. However, $V_{P}^{i}$ is continuous on any subdomain consisting of contract pairs which induce one and the same BE in the agents' game. Moreover, since the dependence of $V_{P}^{i}\left(c^{i}, c^{j}\right)$ upon $c^{j}$ is only through the role $c^{j}$ plays in determining the agents' $\mathrm{BE}$, we have that $\lim _{\epsilon \rightarrow 0} V_{P}^{i}\left(\tilde{c}^{S}(\epsilon), c^{W}\right)$ is equal to $V_{P}^{i}\left(\tilde{c}^{S}(0), c^{I}\right)$ and not equal to $V_{P}^{i}\left(\tilde{c}^{S}(0), c^{W}\right)$.
} 
$1 / U^{\prime}\left(g_{22} \mid \theta_{2}\right)=a / r_{21}$. The $R H S$ of this equation is positive. For the LHS to be positive, we must have $g_{22}>g_{21}$. It remains to be shown that the missing $S S_{1}$ constraint is satisfied. Using Assumption 3 and the fact that $U\left(g_{2 k} \mid \theta_{2}\right)=U\left(g_{1 k} \mid \theta_{2}\right)$ for $k \in\{1,2\}$, we get $U\left(g_{1 k} \mid \theta_{1}\right)>U\left(g_{2 k} \mid \theta_{1}\right)$ for $k \in\{1,2\}$. Therefore, $R_{11} U\left(g_{11} \mid \theta_{1}\right)+R_{12} U\left(g_{12} \mid \theta_{1}\right)>R_{11} U\left(g_{21} \mid \theta_{1}\right)+R_{12} U\left(g_{22} \mid \theta_{1}\right)$. This completes the proof of the first part of the lemma specifying conditions any EBR $\bar{c}^{i}$ to $c^{W}$ satisfies.

Now regarding the existence of an EBR to $c^{W}$ of the desired form, we claim that $\tilde{c}^{S}:[0, \bar{\epsilon}) \rightarrow C^{i}$ introduced in the first part of the proof forms such an EBR to $c^{W}$. We already know that $\left(\tilde{c}^{S}(\epsilon), c^{W}\right)$ induces the recommended separating $\mathrm{BE}$ in the agents' game for all $\epsilon \in(0, \bar{\epsilon})$. By construction of Program $S$, we clearly have $\sum_{i=1}^{2} \sum_{j=1}^{2} r_{i j} \tilde{g}_{i j}=\operatorname{Sup}_{c^{i} \in C^{i}} V_{P}^{i}\left(c^{i}, c^{W}\right)$. Thus, for any $\hat{c}^{i} \in C^{i}$ and $\epsilon \in(0, \bar{\epsilon})$, $V_{p}^{i}\left(\tilde{c}^{S}(\epsilon), c^{W}\right)=r_{11} \tilde{g}_{11}+r_{12} \tilde{g}_{12}+r_{21}\left(\tilde{g}_{21}-\epsilon\right)+r_{22}\left(\tilde{g}_{22}-\epsilon\right)=\sum_{i=1}^{2} \sum_{j=1}^{2} r_{i j} \tilde{g}_{i j}-\left(r_{21}+\right.$ $\left.r_{22}\right) \epsilon>\operatorname{Sup} V_{P}^{i}\left(c^{i}, c^{W}\right)-\epsilon \geq V_{P}^{i}\left(\hat{c}^{i}, c^{W}\right)-\epsilon$. So, $\tilde{c}^{S}(\epsilon)$ is an $\epsilon$-best response of $c^{i} \in C^{i}$

$P^{i}$ to $c^{W}$ of the desired form since the continuity requirement is also clearly met by $\tilde{c}^{S}$.

The Epsilon Best Response to $c^{W}$ defined in Lemma 3 is denoted by $c^{S} .^{22}$ Moreover, for any $\epsilon \in(0, \tilde{\epsilon})$ the associated $\epsilon$-best response to $c^{W}$ is termed an optimal strong comparative contract and denoted by $c^{S}(\epsilon)$. If $P^{j}$ signs $c^{W}$ and $P^{i}$ offers $c^{S}(\epsilon)$, then $A^{i}$ will accept the contract and play the recommended strategy. This follows from condition 8 conjoined with the fact that properties 6 and 10 together imply that playing " $e\left(\theta_{1}\right)=d_{1} ; e\left(\theta_{2}\right)=d_{2}$ " is a dominant strategy for an agent under $c^{S}(0)$ and the unique dominant strategy under $c^{S}(\epsilon)$ with $\epsilon>0$ sufficiently small, and from WFL. Notice that the payouts from the agent under $c^{S}(\epsilon)$ depend on the decisions made by the other agent whatever the behavior of the agent under $c^{S}(\epsilon)$ is $\left(g_{11} \neq g_{12} ; g_{21} \neq g_{22}\right)$. Roughly speaking the structure of payouts under $c^{S}(\epsilon)$ is such that the agent is not only "penalized" for signalling $\theta_{1}$ (by choosing $d_{1}$ ) when the other agent chooses $d_{2}\left[U\left(g_{11} \mid \cdot\right)>\right.$ $U\left(g_{12} \mid \cdot\right)$ ], but also "rewarded" for signalling $\theta_{2}$ (by choosing $d_{2}$ ) as a response to $d_{1}\left[U\left(g_{21}-\epsilon \mid \cdot\right)>U\left(g_{22}-\epsilon \mid \cdot\right)\right]$. The reward for choosing $d_{2}$ when the other agent chooses $d_{1}$ is designed in such a way that an agent who observes $\theta_{2}$ and expects the other agent to always behave as if he had observed $\theta_{1}$ has positive

\footnotetext{
22 The reason for resorting to the notion of an Epsilon Best Response (rather than an ordinary best response) is as follows: Under $c^{S}(0)$ playing the recommended strategy is a dominant strategy for the agent under consideration. Since the same strategy is a best response for the agent under $c^{W}$, the profile " $e^{\alpha}(\cdot)=e^{\beta}(\cdot)=\left(e\left(\theta_{1}\right)=d_{1} ; e\left(\theta_{2}\right)=d_{2}\right)$ " forms a BE under $\left(c^{W}, c^{S}(0)\right)$. However, as before, there is the BE in which $e^{i}(\cdot)=d_{1}$ for all $i \in\{\alpha, \beta\}$ and $\theta^{i} \in \Theta^{i}$. Again, this latter solution leaves each $\theta^{i}$-realization of each agent strictly better off relative to the recommended one. $P^{i}$ can avoid the undesired $\mathrm{BE}$ by sweetening her strategy recommendation just a bit, so as to prevent $A^{i}$ from being indifferent among several dominant strategies. Since $P^{i}$ has a continuous action space, and since an arbitrarily small amount suffices to solve the indifference problem, $P^{i}$ can get arbitrarily close to - but cannot achieve - her best response to $c^{W}$. Note that the alternative version of $W F L_{1}$ defined in Footnote 6 above eliminates the undesired BE under $\left(c^{W}, c^{S}(0)\right)$. So with that version an ordinary best reply would exist and it would be unique.
} 
incentives for obeying the recommendation $f_{2}=d_{2}\left[U\left(g_{21}-\epsilon \mid \theta_{2}\right)>U\left(g_{11} \mid \theta_{2}\right)\right]$. This eliminates the attraction for the agents of jointly adopting strategies other than those intended for them.

Before proceeding it is useful to record a relation between the $V_{P}$ 's for different contract combinations. Since the program leading to $c^{S}(0)$ is more restrictive than that yielding $c^{W}$, but less restrictive than that generating $c^{I}$, we must (in the relevant range of values for $\epsilon$ ) have: $V_{P}\left(c^{W}, c^{I}\right)=V_{P}\left(c^{W}, c^{S}(\epsilon)\right)>$ $V_{P}\left(c^{S}(0), c^{I}\right)>V_{P}\left(c^{I},-\right) .{ }^{23}$ Furthermore, since firstly $\left(c^{W}, c^{W}\right)$ leads to a pooling BE in the agents' game in which each principal's payoff is a constant; secondly, this constant cannot exceed a principal's payoff under an optimal independent pooling contract; and finally, (by the optimality of $c^{I}$ ) a principal's payoff under an optimal independent pooling contract cannot (weakly) exceed that under $c^{I}$, we must have $V_{P}\left(c^{I},-\right)>V_{P}\left(c^{W}, c^{W}\right)$.

The fact that the contract combinations $\left(c^{W}, c^{I}\right)$ and $\left(c^{W}, c^{S}(\epsilon)\right)$ induce (in the releveant range of values for $\epsilon$ ) the same BE in the agents' game implies (together with Lemmas 2 and 3 ) the following result:

Proposition 1. There exist two pure strategy Epsilon Contracting Equlibria in the contract design game among principals. In each of these equilibria one principal plays $c^{W}$ and the other chooses $c^{S}$.

In the pure strategy Epsilon Contracting Equlibria of Proposition $1, P^{\alpha}$ and $P^{\beta}$ act in different ways and have different ex ante payoffs. Since the considered game is perfectly symmetric, it seems natural to look for symmetric equilibria. This leads us to the next result recorded as Proposition 2. Proposition 2 first claims that there exists no symmetric pure strategy ECE and then characterizes one in mixed strategies. To facilitate the understanding of this proposition it might be instructive to start with a simple example in which each principal's pure strategy set is restricted to $\hat{C}^{i}=\left\{c^{I}, c^{S}(\dot{\epsilon}), c^{W}\right\}$, where $\dot{\epsilon}$ is a sufficiently small positive number. From Lemmas 1-3 we know that if $P^{i}$ chooses $c^{I}$, then $P^{j}$ moves to $c^{W}$ which induces $P^{i}$ to play $c^{S}(\dot{\epsilon})$. But then $P^{j}$ sticks to $c^{W}$, which exhausts all the possibilities for a symmetric pure strategy $\mathrm{CE}$ in this simple game. ${ }^{24}$ There is, however, a symmetric equilibrium in mixed strategies: Imagine that $P^{j}$ plays $c^{S}(\dot{\epsilon})$ with probability $\lambda$ and $c^{W}$ with probability $(1-\lambda)$ for some $\lambda \in[0,1]$. Then $P^{i}$, by choosing $c^{S}(\dot{\epsilon})$ gets a payoff of $V_{P}\left(c^{S}(\dot{\epsilon}),-\right)$ irrespective of what contract $c^{j} \in C^{j}$ the principal $P^{j}$ chooses. ${ }^{25}$ By playing $c^{W}, P^{j}$ induces a probability distribution with two possible outcomes: There is a $\lambda$-chance for $\left(c^{W}, c^{S}(\dot{\epsilon})\right)$ to occur, where the agents play the recommended separating $B E$. And there is a chance of $(1-\lambda)$ for $\left(c^{W}, c^{W}\right)$ under which each agent always acts as

\footnotetext{
23 The equality sign in this relation results from the facts that (i) each $P^{i}$, s payoff under a given contract combination does not directly depend upon $c^{j}$, but only indirectly via the behavior of the agents that the combination under consideration generates; and that (ii) for $\epsilon \in\left(0, \phi\left(\theta_{2}, d_{2}\right)-\right.$ $\phi\left(\theta_{2}, d_{1}\right)$, the contract combinations $\left(c^{W}, c^{I}\right)$ and $\left(c^{W}, c^{S}(\epsilon)\right)$ induce the same equilibrium behavior in the agents' game.

${ }^{24}$ Since the pure strategy space of the principals in this simple game is finite there is no need to employ the notion of an ECE.

${ }^{25}$ If $P^{j}$ 's decision domain is $\hat{C}^{j}$, then $P^{i}$ 's ex ante payoff under $c^{S}(\dot{\epsilon})$ is independent of $P^{j}$ 's strategy choice, for all such choices lead to the same BE in the agents' game.
} 
if he had observed $\theta_{1}$. Since $V_{P}\left(c^{W}, c^{S}(\dot{\epsilon})\right)>V_{P}\left(c^{S}(\dot{\epsilon}),-\right)$ and $V_{P}\left(c^{S}(\dot{\epsilon}),-\right)>$ $V_{P}\left(c^{W}, c^{W}\right)$, there is a number $\lambda^{*} \in(0,1)$ such that $\lambda^{*} V_{P}\left(c^{W}, c^{S}(\dot{\epsilon})\right)+(1-$ $\left.\lambda^{*}\right) V_{P}\left(c^{W}, c^{W}\right)=V_{P}\left(c^{S}(\dot{\epsilon}),-\right)$. Now consider the following pair of strategies: "Both principals commit to $c^{S}(\dot{\epsilon})$ with probability $\lambda^{*}$ and to $c^{W}$ with probability $\left(1-\lambda^{*}\right)$ ". Then, since the game is perfectly symmetric and since each $P^{i}$ 's payoff for $c^{S}(\dot{\epsilon})$ is the same as that for $c^{W}$ and these two are strictly higher than $V_{P}\left(c^{I},-\right)$, the range of best responses for any principal (to the opponent's proposed move) is any mixed strategy that assigns zero probability to $c^{I}$. Hence, each principal is ready to play the particular mixed strategy specified above so long as the other does so as well.

The argument remains much the same if we consider the whole set of possible contracts. The main difference is that $c^{W}$ is not optimal any more in an environment in which the combination of $c^{W}$ with the distribution over contracts induced by the strategy played by the other principal yields the $B E$ " $e^{\alpha}(\cdot)=e^{\beta}(\cdot)=\left(e\left(\theta_{1}\right)=d_{1} ; \quad e\left(\theta_{2}\right)=d_{2}\right)$ " with probability $\lambda$ and the $B E$ " $e^{\alpha}(\cdot)=e^{\beta}(\cdot)=\left(e\left(\theta_{1}\right)=d_{1} ; e\left(\theta_{2}\right)=d_{1}\right)$ " with probability $(1-\lambda)$. Thus, a new contract, denoted $c^{\hat{\lambda}}$, is introduced. This contract belongs to a range of "modified weak comparative contracts", indexed by $\lambda \in[0,1]$. Each $c^{\lambda}$ is a close relative of $c^{W}$. Both contain the same decision recommendation " $f_{1}=d_{1} ; f_{2}=d_{2}$ ". They differ, however, in the payout functions: The original weak comparative contract $c^{W}$ chooses the payout schedule so as to maximize $\sum_{k=1}^{2} \sum_{l=1}^{2} r_{k l} g_{k l}$ subject to the requirement that conditional on his private information and the belief that the other agent plays " $e\left(\theta_{1}\right)=d_{1} ; e\left(\theta_{2}\right)=d_{2}$ ", the agent under consideration does not prefer to adopt a strategy other than that designated for him; $c^{W}$ is therefore optimal for a setting in which the BE played by the agents is fully revealing with probability one. The payout schedule in $c^{\lambda}$, on the other hand, is the solution to a modified program which has the same set of constraints but a different objective, namely, it aims to maximize $\lambda \sum_{k=1}^{2} \sum_{l=1}^{2} r_{k l} g_{k l}+(1-\lambda) g_{11}$; thus, $c^{\lambda}$ is the best weak comparative contract for a situation in which there is a chance of $\lambda$ that the BE played by the agents is fully revealing and a chance of $1-\lambda$ that both agents choose the strategy " $e^{i}\left(\theta^{i}\right)=d_{1}\left(\theta^{i} \in \Theta^{i}\right)$ ". In the proof below we show that in the relevant range of values of $\lambda, c^{\lambda}$ has the same qualitative characteristics as $c^{W}$ has. In other words, $c^{\hat{\lambda}}$ exhibits the features listed in Lemma 2. Since we deal here with ECE (rather than just $\mathrm{CE}$ ), where each principal's strategy is a whole family of (simple) contracts $c^{i}(\epsilon)\left(\epsilon \in\left[0, \bar{\epsilon}_{i}\right)\right)$, we will, of course, have one modified weak comparative contract $c^{\lambda(\epsilon)}$ for each $\epsilon \in\left[0, \bar{\epsilon}_{i}\right)$.

Proposition 2. There is no symmetric pure strategy ECE in the perfectly symmetric game among principals. In a symmetric ECE in mixed strategies each principal plays $c^{S}(\epsilon)$ with probability $\hat{\lambda}(\epsilon) \in(0,1)$ and $c^{\hat{\lambda}(\epsilon)}$ with probability $1-\hat{\lambda}(\epsilon)$.

Proof. NONEXISTENCE of a Symmetric Pure-Strategy ECE. Suppose that there is an $\operatorname{ECE}\left(\bar{c}^{\alpha}, \bar{c}^{\beta}\right)$ with $\bar{c}^{\alpha}=\bar{c}^{\beta}$, where $\bar{c}^{\alpha}:[0, \bar{\epsilon}) \rightarrow C^{\alpha}$ and $\bar{c}^{\beta}:[0, \bar{\epsilon}) \rightarrow C^{\beta}$ for some $\bar{\epsilon}>0$. We will show that this supposition leads to a contradiction for each possible BE the contract pair $\left(\bar{c}^{\alpha}, \bar{c}^{\beta}\right)$ may induce in the agents' game. For each $\epsilon \in[0, \bar{\epsilon})$, set $\bar{c}^{i}(\epsilon)=\left(\bar{f}_{1}, \bar{f}_{2}, \bar{g}_{11}(\epsilon), \bar{g}_{12}(\epsilon), \bar{g}_{21}(\epsilon), \bar{g}_{22}(\epsilon)\right)(i \in\{\alpha, \beta\})$. 
Case 1. First assume that, for each $n \in \mathbf{N}$, there exists some $\epsilon_{n} \in$ $\left(0, \frac{1}{n}\right) \cap(0, \bar{\epsilon})$ such that each agent's equilibrium strategy in the agents' game under $\left(\bar{c}^{\alpha}\left(\epsilon_{n}\right), \bar{c}^{\beta}\left(\epsilon_{n}\right)\right)$ is fully pooling, i.e. of the form " $e\left(\theta_{1}\right)=e\left(\theta_{2}\right)=d_{k}$ " for some $k \in\{0,1\}$. Now it follows from the proof of Lemma 1 that $V_{P}^{\alpha}\left(\bar{c}^{\alpha}\left(\epsilon_{n}\right), \bar{c}^{\beta}\left(\epsilon_{n}\right)\right) \leq \phi\left(\theta_{1}, d_{1}\right)$ for each $n \in \mathbf{N}$. Since $\phi\left(\theta_{1}, d_{1}\right)<V_{P}^{\alpha}\left(c^{I},-\right)$, if we choose $\bar{n} \in \mathbf{N}$ with $\frac{1}{\bar{n}}<V_{P}^{\alpha}\left(c^{I},-\right)-\phi\left(\theta_{1}, d_{1}\right)$, we get that $V_{P}^{\alpha}\left(c^{I}, \bar{c}^{\beta}\left(\epsilon_{\bar{n}}\right)\right)=$ $V_{P}^{\alpha}\left(c^{I},-\right)>V_{P}^{\alpha}\left(\bar{c}^{\alpha}\left(\epsilon_{\bar{n}}\right), \bar{c}^{\beta}\left(\epsilon_{\bar{n}}\right)\right)+\epsilon_{\bar{n}}$, in contradiction with $\bar{c}^{\alpha}\left(\epsilon_{\bar{n}}\right)$ being an $\epsilon_{\bar{n}}{ }^{-}$ best response to $\bar{c}^{\beta}\left(\epsilon_{\bar{n}}\right)$. Therefore, for all sufficiently small $\epsilon$ in $(0, \bar{\epsilon})$, the $\mathrm{BE}$ in the agents' game cannot consist of pooling strategies. Without loss of generality, we can assume that this is the case for all $\epsilon$ in $(0, \bar{\epsilon})$.

Case 2. Now suppose that, for each $n \in \mathbf{N}$, there exists some $\epsilon_{n} \in\left(0, \frac{1}{n}\right) \cap$ $(0, \bar{\epsilon})$ such that the strategies at the BE of the induced agents' game are fully revealing. It is easy to see that any revealing strategy combination $\left(e^{\alpha}, e^{\beta}\right)$ except for the one where $e^{i}\left(\theta_{k}\right)=d_{k}$ for all $i \in\{\alpha, \beta\}, k \in\{1,2\}$ cannot be induced under any $\operatorname{ECE}\left(\bar{c}^{\alpha}(\epsilon), \bar{c}^{\beta}(\epsilon)\right)$ when $\epsilon$ is sufficiently small which we can without loss of generality assume to be already the case for all $\epsilon \in(0, \bar{\epsilon})$. But then $\bar{g}_{k l}\left(\epsilon_{n}\right)$ $(k, l \in\{1,2\}, n \in \mathbf{N})$ satisfies $S S_{1}, S S_{2}, I R_{1}, I R_{2}$ and $\left(a_{2}\right)$. By continuity of $\bar{g}_{k l}$ and $U$, this means that $\bar{g}_{k l}(0)\left(=\lim _{n \rightarrow \infty} \bar{g}_{k l}\left(\epsilon_{n}\right)\right)$ satisfies $S S_{1}, S S_{2}, I R_{1}, I R_{2}$ and $\left(a_{2}^{\prime}\right)$. But we know from the proof of Lemma 3 that then $\left(\bar{g}_{11}(0), \bar{g}_{12}(0), \bar{g}_{21}(0), \bar{g}_{22}(0)\right)$ is a solution to Program $S$.

Now since $\left(c^{W}, \bar{c}^{\beta}(\epsilon)\right)$ induces the same separating $\mathrm{BE}$ as $\left(c^{W}, c^{S}(\epsilon)\right)$ for all $\epsilon \in(0, \bar{\epsilon}), c^{W}$ is a best response of $P^{\alpha}$ to $\bar{c}^{\beta}(\epsilon)$ for each such $\epsilon$ which, in particular, implies that

$$
V_{P}^{\alpha}\left(c^{W}, \bar{c}^{\beta}(\epsilon)\right)>V_{P}^{\alpha}\left(\bar{c}^{\alpha}(0), c^{I}\right),
$$

where $V_{P}^{\alpha}\left(c^{W}, \bar{c}^{\beta}(\epsilon)\right)$ is constant on $(0, \bar{\epsilon})$. Set $\eta=\frac{1}{2}\left(V_{P}^{\alpha}\left(c^{W}, \bar{c}^{\beta}(\epsilon)\right)-V_{P}^{\alpha}\left(\bar{c}^{\alpha}(0)\right.\right.$, $\left.\left.c^{I}\right)\right)>0$. Since $\bar{g}_{k l}$ is continuous on $[0, \bar{\epsilon})$, there is some $\delta>0$ such that $\left|\bar{g}_{k l}\left(\epsilon^{\prime}\right)-\bar{g}_{k l}(0)\right|<\eta$ for all $\epsilon^{\prime} \in(0, \delta)$ and $k, l \in\{1,2\}$. Now choose $n \in \mathbf{N}$ such that $\frac{1}{n}<\operatorname{Min}\{\eta, \delta\}$. Then $\epsilon_{n}<\eta$ and $\epsilon_{n}<\delta$. Note that $\mid V_{P}^{\alpha}\left(\bar{c}^{\alpha}(0), c^{I}\right)-$ $V_{P}^{\alpha}\left(\bar{c}^{\alpha}\left(\epsilon_{n}\right), \bar{c}^{\beta}\left(\epsilon_{n}\right)\right)|=| \sum_{k=1}^{2} \sum_{l=1}^{2} r_{k l}\left(\bar{g}_{k l}(0)-\bar{g}_{k l}\left(\epsilon_{n}\right)\right)\left|\leq \sum_{k=1}^{2} \sum_{l=1}^{2} r_{k l}\right| \bar{g}_{k l}(0)-\bar{g}_{k l}\left(\epsilon_{n}\right) \mid<$ $\eta$. Combining this with the definition of $\eta$, we get that

$$
V_{P}^{\alpha}\left(c^{W}, \bar{c}^{\beta}\left(\epsilon_{n}\right)\right)-V_{P}^{\alpha}\left(\bar{c}^{\alpha}\left(\epsilon_{n}\right), \bar{c}^{\beta}\left(\epsilon_{n}\right)\right)>\eta>\epsilon_{n},
$$

contradicting that $\bar{c}^{\alpha}\left(\epsilon_{n}\right)$ is an $\epsilon_{n}$-best response to $\bar{c}^{\beta}\left(\epsilon_{n}\right)$. Like in Case 1 , this allows to conclude that the BE in the agents' game cannot consist of separating strategies for any $\epsilon \in(0, \bar{\epsilon})$.

Case 3: Finally we will consider the case where, for each $n \in \mathbf{N}$, there exists some $\epsilon_{n} \in\left(0, \frac{1}{n}\right) \cap(0, \bar{\epsilon})$ such that the agents' strategies at the induced $\mathrm{BE}$ are mixed ones where the probability assigned to at least one strategy lies in $(0,1)$. The proof that this cannot be the case either is delegated to the Appendix. Thus, assuming that the BE in the agents' game are symmetric by focal point considerations, this completes the proof of the nonexistence of symmetric pure strategy ECE. 
EXISTENCE of a Symmetric Epsilon Contracting Equilibrium in Mixed Strategies. The proof is devided into four steps.

Step 1. In the first step we introduce a range of modified weak comparative contracts, each indexed by $\lambda \in[0,1]$. Each contract in this family recommends the strategy " $e\left(\theta_{1}\right)=d_{1} ; e\left(\theta_{2}\right)=d_{2}$ "; the payout schedule $\left(g_{11}, g_{12}, g_{21}, g_{22}\right)$ in $c^{\lambda}$ is chosen in order to maximize $\lambda \sum_{k=1}^{2} \sum_{l=1}^{2} r_{k l} g_{k l}+(1-\lambda) g_{11}$ subject to $S S_{1}$, $S S_{2}, I R_{1}$, and $I R_{2}$ (as defined in the proof of Lemma 2) and to $I R_{x}$ defined as the constraint $U\left(g_{11} \mid \theta_{1}\right) \geq 0$. We denote this program by $W(\lambda)$ and the maximal value of the objective function for a given $\lambda$ by $M(\lambda)$.

Step 2. Next we search, for each $\epsilon \in(0, \bar{\epsilon})$, with $\bar{\epsilon}=\left(r_{21}+r_{22}\right)\left[\phi\left(\theta_{2}, d_{2}\right)-\right.$ $\left.\phi\left(\theta_{2}, d_{1}\right)\right]$, for a number $\lambda(\epsilon) \in(0,1)$ such that $M(\lambda(\epsilon))=V_{P}\left(c^{S}(\epsilon), c^{W}\right)$. First we have to show that such a $\lambda$ exists. This is easily verified: If $\lambda=1$, the objective in the program $W(\lambda)$ is the same as that in the program $S$. Since the optimal solution to $S$ is feasible as a solution to $W(\lambda)$, but not optimal, we have $M(1) V_{P}\left(c^{S}(0), c^{I}\right)>V_{P}\left(c^{S}(\epsilon), c^{I}\right)=V_{P}\left(c^{S}(\epsilon), c^{W}\right) .{ }^{26}$ If $\lambda=0$, the program $W(\lambda)$ boils down to the problem of designing a payout schedule for an optimal independent pooling contract in which $f(\cdot)=d_{1}$ for each $\theta$. From the arguments in the proof of Lemma 1, we know that the ex ante payoff of a principal under such a contract is $\phi\left(\theta_{1}, d_{1}\right)$. Since, for $\epsilon<\left(r_{21}+r_{22}\right)\left[\phi\left(\theta_{2}, d_{2}\right)-\phi\left(\theta_{2}, d_{1}\right)\right]$, $V_{P}\left(c^{S}(\epsilon), c^{W}\right)>\phi\left(\theta_{1}, d_{1}\right)$ we have $V_{P}\left(c^{S}(\epsilon), c^{W}\right)>M(0)$. To complete the proof for the existence it remains to be demonstrated that $M(\lambda)$ is continuous. This follows from the Theorem of the Maximum of Berge (1963). To guarantee uniqueness, it will suffice to also show that $M(\lambda)$ is strictly increasing in the relevant range. To see this, first note that the component of the objective function that relates to $1-\lambda$ can never exceed $\phi\left(\theta_{1}, d_{1}\right)<V_{P}\left(c^{I},-\right)$. Thus, in that range of $\lambda$ where $M(\lambda) \geq V_{P}\left(c^{I},-\right)$, the component of the objective function that relates to $\lambda$ must strictly exceed the component that relates to $1-\lambda$. Since the $\lambda$-component obtains more and the $(1-\lambda)$-component less weight if $\lambda$ increases, and since it is always possible not to adapt the $\lambda$ - and $(1-\lambda)$-parts, $M(\lambda)$ must increase in $\lambda$ in this range. We therefore conclude: For each $\epsilon \in(0, \bar{\epsilon})$, with $\bar{\epsilon}=\left(r_{21}+r_{22}\right)\left[\phi\left(\theta_{2}, d_{2}\right)-\phi\left(\theta_{2}, d_{1}\right)\right]$, the condition $M(\lambda)=V_{P}\left(c^{S}(\epsilon), c^{W}\right)$ defines a unique $\lambda$ in $(0,1)$. We denote this $\lambda$ by $\hat{\lambda}(\epsilon)$ and the accompanying modified weak comparative contract by $c^{\hat{\lambda}(\epsilon)}$.

Step 3. Now we claim that the payout schedule in $c^{\hat{\lambda}(\epsilon)}$ has the properties listed in Lemma 2. To show this, we examine a relaxed version of $W(\lambda)$ in which $S S_{1}$ and $I R_{x}$ are not included and go step by step the same way as in the proof of Lemma 2. All the arguments continue to hold, except one: $g_{12}>g_{11}$ cannot be proved by analyzing the $F O C$ s. Here the proof is by contradiction: Suppose that $g_{21}=g_{22}$ but $g_{11} \geq g_{12}$. Then we could define a new payout schedule in which $g_{11}$ and $g_{12}$ are replaced by $\bar{g}_{1}$, where $\bar{g}_{1}$ is such that $U\left(\bar{g}_{1} \mid \theta_{1}\right)=R_{11} U\left(g_{11} \mid \theta_{1}\right)+$ $R_{12} U\left(g_{12} \mid \theta_{1}\right)$. The definition of $\bar{g}_{1}$ guarantees that $I R_{1}$ is maintained. That $S S_{2}$ is satisfied can be seen from the sequence $R_{21} U\left(g_{21} \mid \theta_{2}\right)+R_{22} U\left(g_{22} \mid \theta_{2}\right) \geq$

\footnotetext{
26 To see this, observe that the programs $W, W(1)$ and $S$ differ only in the constraints. The least restricted program is $W$. Since the optimal solution to $W$ has $g_{12}>g_{11}$, it is also a solution to $W(1)$. Since it violates $\left(a_{2}\right)$ - as defined in the proof of Lemma 3 - it is not a solution to $S$.
} 
$R_{21} U\left(g_{11} \mid \theta_{2}\right)+R_{22} U\left(g_{12} \mid \theta_{2}\right) \geq R_{11} U\left(g_{11} \mid \theta_{2}\right)+R_{12} U\left(g_{12} \mid \theta_{2}\right) \geq U\left(\bar{g}_{1} \mid \theta_{2}\right)$, where the first inequality follows from the original payout schedule being feasible, the second from $g_{11} \geq g_{12}$ and $R_{11}>R_{21}$, and the last from the definition of $\bar{g}_{1}$ and NIARA. Moreover, since $U(\cdot)$ is strictly increasing and concave, $\bar{g}_{1} \geq$ $R_{11} g_{11}+R_{12} g_{12}$, so that that component of the objective function that relates to $\lambda$ is improved. The new payout schedule is a feasible solution to the program defined in the proof of Lemma 1. The maximal value of the objective function in this program is $V_{P}\left(c^{I},-\right)$. So under the original payout schedule the value of the $\lambda$-part of the objective function in $W(\lambda)$ must have been (weakly) less than $V_{P}\left(c^{I},-\right)$. By the arguments in Step 2 the value of the $(1-\lambda)$-part of the objective function in $W(\lambda)$ can never exceed $V_{P}\left(c^{I},-\right)$. But then the sum of the two parts could not have been $M(\hat{\lambda}(\epsilon))=V_{P}\left(c^{S}(\epsilon), c^{W}\right)>V_{P}\left(c^{I},-\right)$. It remains only to be verified that at the solution to the relaxed program $S S_{1}$ and $I R_{x}$ are satisfied. The argument for $S S_{1}$ is the same as that in the proof of Lemma 2 . And from $U\left(g_{11} \mid \cdot\right)>U\left(g_{12} \mid \cdot\right)$ and $I R_{1}$ it follows that $U\left(g_{11} \mid \theta_{1}\right)>0$, so that $I R_{x}$ holds as a strict inequality.

Step 4. We now complete the proof by showing that $\left(\bar{\gamma}^{\alpha}, \bar{\gamma}^{\beta}\right)$ is a mixed strategy ECE with $\bar{\gamma}^{\alpha}=\bar{\gamma}^{\beta}=\left(\left(c^{S}, c^{\hat{\lambda}}\right) ;\{\hat{\pi}[\epsilon] \mid \epsilon \in(0, \bar{\epsilon})\}\right)$, where $c^{\hat{\lambda}}(\epsilon)=c^{\hat{\lambda}(\epsilon)}$, $\hat{\pi}[\epsilon]\left(c^{S}(\epsilon)\right)=\hat{\lambda}(\epsilon)$ and $\hat{\pi}[\epsilon]\left(c^{\hat{\lambda}}(\epsilon)\right)=1-\hat{\lambda}(\epsilon)$ for each $\epsilon \in[0, \bar{\epsilon})$. Let $\{i, j\}=$ $\{\alpha, \beta\}$, and take any $\epsilon \in(0, \bar{\epsilon})$. Now $V_{P}^{j}\left(c^{S}(\epsilon), \bar{\gamma}^{i}(\epsilon)\right)=\hat{\lambda}(\epsilon) V_{P}^{j}\left(c^{S}(\epsilon), c^{S}(\epsilon)\right)+$ $(1-\hat{\lambda}(\epsilon)) V_{P}^{j}\left(c^{S}(\epsilon), c^{\hat{\lambda}(\epsilon)}\right)$. Since $c^{\hat{\lambda}(\epsilon)}$ satisfies the properties of Lemma 2, it follows directly from the proof Lemma 3 that the same BE, where both agents play " $e\left(\theta_{1}\right)=d_{1}, e\left(\theta_{2}\right)=d_{2}$ ", is induced under both $\left(c^{S}(\epsilon), c^{W}\right)$ and $\left(c^{S}(\epsilon), c^{\hat{\lambda}(\epsilon)}\right)$. Moreover, by construction of $c^{S}$, the same equilibrium strategies are induced as the unique dominant strategies of both agents under $\left(c^{S}(\epsilon), c^{S}(\epsilon)\right)$. Thus, $V_{P}^{j}\left(c^{S}(\epsilon), c^{S}(\epsilon)\right)=V_{P}^{j}\left(c^{S}(\epsilon), c^{\hat{\lambda}(\epsilon)}\right)=V_{P}^{j}\left(c^{S}(\epsilon), c^{W}\right)$, so that $V_{P}^{j}\left(c^{S}(\epsilon), \bar{\gamma}^{i}(\epsilon)\right)=$ $V_{P}^{j}\left(c^{S}(\epsilon), c^{W}\right)$.

To compute $V_{P}^{j}\left(c^{\hat{\lambda}(\epsilon)}, \bar{\gamma}^{i}(\epsilon)\right)$, first note that the separating BE, where both agents play " $e\left(\theta_{1}\right)=d_{1}, e\left(\theta_{2}\right)=d_{2}$ ", is induced under $\left(c^{\hat{\lambda}(\epsilon)}, c^{S}(\epsilon)\right)$; and the pooling $\mathrm{BE}$, where both agents play " $e\left(\theta_{1}\right)=e\left(\theta_{2}\right)=d_{1}$ ", is induced un$\operatorname{der}\left(c^{\hat{\lambda}(\epsilon)}, c^{\hat{\lambda}(\epsilon)}\right)$ again since $c^{\hat{\lambda}(\epsilon)}$ shares all the characteristics of $c^{W}$ listed in Lemma 2. Denoting the payout schedule in $c^{\hat{\lambda}(\epsilon)}$ simply by $\left(g_{11}, g_{12}, g_{21}, g_{22}\right)$, we conclude that $V_{P}^{j}=\left(c^{\hat{\lambda}(\epsilon)}, \bar{\gamma}^{i}(\epsilon)\right)=\hat{\lambda}(\epsilon) \sum_{k=1}^{2} \sum_{l=1}^{2} r_{k l} g_{k l}+(1-\hat{\lambda}(\epsilon)) g_{11}$, which is equal to $M(\hat{\lambda}(\epsilon))$ by definition of $c^{\hat{\lambda}(\epsilon)}$. Moreover, $M(\hat{\lambda}(\epsilon))=V_{P}^{j}\left(c^{S}(\epsilon), c^{W}\right)$ by the choice of $\hat{\lambda}(\epsilon)$. Thus, $P^{j}$ is indifferent between responding to $\bar{\gamma}^{i}(\epsilon)$ by playing $c^{S}(\epsilon)$ or $c^{\hat{\lambda}(\epsilon)}$, and $V_{P}^{j}\left(\bar{\gamma}^{j}(\epsilon), \bar{\gamma}^{i}(\epsilon)\right)=V_{P}^{j}\left(c^{S}(\epsilon), c^{W}\right)$ for all $\epsilon \in(0, \bar{\epsilon})$.

Now take any $\bar{c}_{1}^{j}, \ldots, \bar{c}_{m}^{j}:[0, \bar{\epsilon}) \rightarrow C^{j}$ and, for each $\epsilon \in[0, \bar{\epsilon})$, let $\bar{\pi}^{j}[\epsilon]$ be a probability distribution on $\left\{\bar{c}_{1}^{j}(\epsilon), \ldots, \bar{c}_{m}^{j}(\epsilon)\right\}$. Pick any $\epsilon \in(0, \bar{\epsilon})$ and any $\bar{c}_{t}^{j}(\epsilon)$. We will consider different cases according to what Bayesian equilibria are induced in the agents' game under the contract pairs $\left(\bar{c}_{t}^{j}(\epsilon), c^{S}(\epsilon)\right)$ and $\left(\bar{c}_{t}^{j}(\epsilon), c^{\hat{\lambda}(\epsilon)}\right)$. In doing so, we will confine ourselves to the strategies " $e\left(\theta_{1}\right)=d_{1} ; e\left(\theta_{2}\right)=d_{2}$ " and " $e\left(\theta_{1}\right)=e\left(\theta_{2}\right)=d_{1}$ " for both agents without loss of generality since our aim is to show that $\bar{\gamma}^{j}(\epsilon)$ is an $\epsilon$-best response to $\bar{\gamma}^{i}(\epsilon)$. 
First consider the case where the BE under $\left(\bar{c}_{t}^{j}(\epsilon), c^{S}(\epsilon)\right)$ is separating while the BE under $\left(\bar{c}_{t}^{j}(\epsilon), c^{\hat{\lambda}(\epsilon)}\right)$ is pooling. Then the payout schedule in $\bar{c}_{t}^{j}(\epsilon)$ will satisfy the constraints in $W(\hat{\lambda}(\epsilon))$ and, by definition of $c^{\hat{\lambda}(\epsilon)}, V_{P}^{j}\left(\bar{c}_{t}^{j}(\epsilon), \bar{\gamma}^{i}(\epsilon)\right) \leq$ $V_{P}^{j}\left(c^{S}(\epsilon), c^{W}\right)=V_{P}^{j}\left(\bar{\gamma}^{j}(\epsilon), \bar{\gamma}^{i}(\epsilon)\right)$. Now assume that $\bar{c}_{t}^{j}(\epsilon)$ is such that both $\left(c_{t}^{j}(\epsilon), c^{S}(\epsilon)\right)$ and $\left(c_{t}^{j}(\epsilon), c^{\hat{\lambda}(\epsilon)}\right)$ lead to the separating BE in the agents' game. Since $c^{\hat{\lambda}(\epsilon)}$ possesses all the properties listed in Lemma 2 and $\left(\bar{c}_{t}^{j}(\epsilon), c^{\hat{\lambda}(\epsilon)}\right)$ induces the separating BE, the payout schedule $\left(g_{11}, g_{12}, g_{21}, g_{22}\right)$ in $\bar{c}_{t}^{j}(\epsilon)$ satisfies all the constraints in Program $S$. So,

$$
\begin{aligned}
V_{P}^{j}\left(\bar{c}_{t}^{j}(\epsilon), \bar{\gamma}^{i}(\epsilon)\right) & =\hat{\lambda}(\epsilon) V_{P}^{j}\left(\bar{c}_{t}^{j}(\epsilon), c^{S}(\epsilon)\right)+(1-\hat{\lambda}(\epsilon)) V_{P}^{j}\left(\bar{c}_{t}^{j}(\epsilon), c^{\hat{\lambda}(\epsilon)}\right) \\
& =\sum_{k=1}^{2} \sum_{l=1}^{2} r_{k l} g_{k l} \leq V_{P}^{j}\left(c^{S}(0), c^{I}\right) \leq V_{P}^{j}\left(c^{S}(\epsilon), c^{W}\right)+\epsilon \\
& =V_{P}^{j}\left(\bar{\gamma}^{j}(\epsilon), \bar{\gamma}^{i}(\epsilon)\right)+\epsilon,
\end{aligned}
$$

by definition of $c^{S}$. Having dealt with the typical two cases, one similarly shows that the same inequality holds for all possible BE which may arise in the agents' game. But then

$$
\sum_{t=1}^{m} \bar{\pi}^{j}[\epsilon]\left(\bar{c}_{t}^{j}(\epsilon)\right) V_{P}^{j}\left(\bar{c}_{t}^{j}(\epsilon), \bar{\gamma}^{i}(\epsilon)\right) \leq V_{P}^{j}\left(\bar{\gamma}^{j}(\epsilon), \bar{\gamma}^{i}(\epsilon)\right)+\epsilon,
$$

implying that $\bar{\gamma}^{j}(\epsilon)$ is a mixed strategy $\epsilon$-best response to $\bar{\gamma}^{i}(\epsilon)$ for all $\epsilon \in(0, \bar{\epsilon})$, or equivalently, that $\bar{\gamma}^{j}$ is a mixed strategy Epsilon Best Response to $\bar{\gamma}^{i}$. Finally, since $\bar{\gamma}^{i}=\bar{\gamma}^{j}$, we conclude that $\left(\bar{\gamma}^{\alpha}, \bar{\gamma}^{\beta}\right)$ is a symmetric mixed strategy ECE. $\square$

In the ECE of Proposition 2 the lack of coordination among principals generates various inefficiencies: First, there is a chance of $(\hat{\lambda}(\epsilon))^{2}$ at the contract combination $\left(c^{S}(\epsilon), c^{S}(\epsilon)\right)$. In this realization there is "excess control": Both principals employ a contract with a high-powered payout schedule although a single high-powered incentive scheme (when combined with a weak comparative contract) would suffice to induce both agents to obey the decision recommendation " $f_{1}=d_{1} ; f_{2}=d_{2}$ ". In this realization one of the $P^{i}$, s could obtain strict gains by switching to $c^{W}$. Secondly, there is a chance of $\hat{\lambda}(\epsilon)(1-\hat{\lambda}(\epsilon))$ at each of the two mirror-image pairs $\left(c^{\hat{\lambda}(\epsilon)}, c^{S}(\epsilon)\right)$ and $\left(c^{S}(\epsilon), c^{\hat{\lambda}(\epsilon)}\right)$. These institutional structures are also not efficient relative to incentive constraints: Although $c^{\hat{\lambda}(\epsilon)}$ is (ex ante) optimal for probabilistic beliefs about the contract chosen by the second principal, it is not (ex post) optimal once this uncertainty has been resolved. The principal choosing $c^{\hat{\lambda}(\epsilon)}$ could obtain strict gains by redesigning her contract to $c^{W}$. Finally, there is a chance of $(1-\hat{\lambda}(\epsilon))^{2}$ at $\left(c^{\hat{\lambda}(\epsilon)}, c^{\hat{\lambda}(\epsilon)}\right)$. Under this contract combination the agents play " $e^{\alpha}(\cdot)=e^{\beta}(\cdot)=\left(e\left(\theta_{1}\right)=d_{1} ; e\left(\theta_{2}\right)=d_{1}\right)$ " and some of the profits are dissipated wastefully since Stage 2 decisions are not optimally tailored to the environment. Both principals would be strictly better off with a more powerful payoff schedule in one of the firms.

The explanation for the inefficiencies in the design of incentives implied by Proposition 2 is basically the same as that for other coordination failure results in 
the economic literature. The key observation is that the presence of externalities can lead to the existence of multiple non-cooperative equilibria. The multiplicity of equilibria creates a demand for coordination. With uncoordinated maximization an industry or economy can get stuck at an inefficient equilibrium even though a superior non-cooperative solution exists. In the inefficient equilibrium profitable opportunities from an overall change in strategies (from a coordinated change in institutional design) remain unrealized. ${ }^{27}$

Of course, inefficiencies of the coordination failure type do not arise in traditional principal-agent models in which a single authority designs the overall institutional network. They are also impossible in a decentralized setting when each principal acts as if she were alone (with her agents) on a desert island. These settings are, however, not very realistic: In the real world institutions are designed not so much to perform perfectly in isolation but rather to do well in an institutional network. Consequently, interdependences between different agencies arise and with them a demand for coordination.

Before proceeding it is important to recall that Proposition 1 characterizes two pure-strategy but asymmetric Epsilon Contracting Equlibria. The coordination failure result of Proposition 2 should therefore best be interpreted as indicating a possibility of inefficiency and not as suggesting inefficiency with probability one. Finally notice that the mixed strategy equilibrium of Proposition 2 can be converted into a pure-strategy equilibrium by introducing an additional source of nature-created uncertainty. ${ }^{28}$ There is therefore no a priori reason for discrediting this equilibrium with the argument that randomized strategies are a convenient game-theoretic construction intended to prove rather nice equilibrium-existence results, but not serious predictors of economic activity.

\section{Related work}

The pioneering work dealing with models in which several principals interact in designing contracts for their agents is due to Myerson (1982). Although his setup is similar to ours in many respects, there are also some important differences between the two models. For one thing, Myerson (1982) considers a fairly general model covering both hidden action and hidden information problems while we focus here on a special hidden information problem. In fact, in our model it is exactly the observability of both agents' actions by all the principals which enables them to make their contracts dependent not only upon their own agent's but also upon the other's agent's actions.

\footnotetext{
27 Note that the contract-proposing game among principals is very much in the spirit of the "Grabthe-Dollar" game. In fact, if each $P^{i}$ is constrained to pick an element in $C^{\alpha}=C^{\beta}=\left\{c^{S}(\epsilon), c^{W}\right\}$ one gets a game which is "isomorphic" to the static version of this game. Dynamic versions of the Grab-the-Dollar game have been used in IO to discuss coordination problems in markets in which two or more firms have to decide whether and when to build a new plant or to adopt a new technology when the market is big enough to support only one such addition.

28 The idea that randomized strategies can be thought off as pure strategies in (slightly) perturbed games of incomplete information forms the basis of the "purification theorems" of Harsanyi (1973) and Aumann et al. (1983).
} 
Another crucial difference is concerned with the time and information structures reflected by the respective extensive form games constructed in the two models as well as the associated equilibrium notions employed in analyzing these. The counterpart of our notion of an Epsilon Contracting Equilibrium in Myerson (1982) is what he calls a Principals' Equilibrium. In a framework within which both equilibrium concepts are well-defined, Principals' Equilibria may not exist, whereas a (zero-) Contracting Equilibrium does exist, as will be exemplified below. The main reason for this is that, in Myerson's (1982) model, the agents know only the relevant probability distributions over contracts when upon to move if the principals play nondegenerate mixed strategies. This simply means that Myerson's (1982) game cannot be represented as the mixed extension of a finite extensive form game; it is rather a genuinely infinite game although all the basic sets of states, decisions or (pure) contracts are finite. The extensive form game in the present paper is, however, such that the agents, i.e. the players of the second stage game, move only after having perfectly observed the outcome of the first stage, i.e. the contracts chosen by both of the principals. How this difference leads to the absence of Principals' Equilibria in some games which own Epsilon Contracting Equilibria can best be seen by considering Myerson's (1982) example for his nonexistence results.

Myerson (1982) considers a model with finitely many principals, each endowed with her own finite set of agents. The sets of states of agents' precontractual information and their action spaces are all finite, just as also are the principals' decision domains. We can now easily translate Myerson's (1982) particular nonexistence example which fits our framework fairly well into our notation: There are two principals $\left(P^{\alpha}\right.$ and $\left.P^{\beta}\right)$, each with one agent $\left(A^{\alpha}\right.$ and $A^{\beta}$, respectively). The two possible realizations of the agent's parameters of precontractual information are the same and given by $\Theta^{\alpha}=\Theta^{\beta}=\left\{\theta_{1}, \theta_{2}\right\}$. They have equal probability, and the two agents' parameter realizations are independent which in our notation amounts to $r_{11}^{i}=r_{12}^{i}=r_{21}^{i}=r_{22}^{i}=\frac{1}{4}$ for each $i \in\{\alpha, \beta\}$. Each principal's underlying decision domain over which randomization is made consists of two elements, a separating contract $c^{S E}$ and a pooling contract $c^{P O}$, so that $C^{\alpha}=C^{\beta}=\left\{c^{S E}, c^{P O}\right\}{ }^{29}$ Similarly, the agents' decision domains also consist of two elements each with $D^{\alpha}=D^{\beta}=\left\{d_{1}, d_{2}\right\}$. For each $i \in\{\alpha, \beta\}$, the payoff $V_{P}^{i}$ to $P^{i}$ depends upon $A^{i}$,s and $P^{i}$ 's decisions along with the realization of $A^{i}$,s state of precontractual private information, while the payoff $V_{A}^{i}$ to $A^{i}$ is also dependent upon the other principal's decision in addition to these. More specifically, the payoffs are given through the matrix

\begin{tabular}{l|cccc}
\hline$V_{P}^{i}, V_{A}^{i}$ & $\left(\theta_{1}, d_{1}\right)$ & $\left(\theta_{1}, d_{2}\right)$ & $\left(\theta_{2}, d_{1}\right)$ & $\left(\theta_{2}, d_{2}\right)$ \\
\hline$c^{S E}$ & 6,1 & $0, z^{i}$ & $0, z^{i}$ & 6,1 \\
$c^{P O}$ & 5,0 & 5,0 & 5,0 & 5,0
\end{tabular}

\footnotetext{
${ }^{29}$ Strictly speaking, a principal's pure strategy set in Myerson (1982) has six elements if these are to be expressed in the format of the present paper's contracts. However, there is no loss of generality in confining our attention only to $c^{S E}$ and $c^{P O}$ for our purposes here, since the other four pure strategies are strictly dominated under Myerson's (1982) assumptions.
} 
where $z^{\alpha}=2$ if $P^{\beta}$ chooses $c^{S E}, z^{\alpha}=1$ if $P^{\beta}$ chooses $c^{P O} ; z^{\beta}=1$ if $P^{\alpha}$ chooses $c^{S E}$, and $z^{\beta}=2$ if $P^{\alpha}$ chooses $c^{P O}$. Myerson (1982) concentrates on incentive compatible mechanisms leading to truth-telling equilibria. This, in turn, entails the implicit assumption that agents maximize lexicographically, first their own utilities and, in case of indifference, those of their respective principals.

Now suppose that a Principals' Equilibrium exists in this game. First consider the case where $P^{\alpha}$ plays $c^{S E}$ with probability 1 at this equilibrium. Now $z^{\beta}=1$ for sure and thus, by playing $c^{S E}$ with probability $1, P^{\beta}$ can take advantage of the lexicographic nature of her agent's preferences, for this pair of contracts will induce $A^{\beta}$ to play " $e\left(\theta_{1}\right)=d_{1} ; e\left(\theta_{2}\right)=d_{2}$ " with probability 1 , leading to a payoff of 6 for sure for $P^{\beta}$. This, however, makes now $z^{\alpha}=2$ for sure, inducing the behaviour " $e\left(\theta_{1}\right)=d_{2} ; e\left(\theta_{2}\right)=d_{1}$ " with probability 1 on the part of $A^{\alpha}$, which results in a payoff of 0 for sure to $P^{\alpha}$ who could have guaranteed a payoff of 5 for herself by simply choosing $c^{P O}$ with probability 1 . Thus, if a Principals' Equilibrium exists at all, then $P^{\alpha}$ must be playing $c^{P O}$ with positive probability at that equilibrium. Then, however, $z^{\beta}=2$ with positive probability, inducing $A^{\beta}$ to play " $e\left(\theta_{1}\right)=d_{2} ; e\left(\theta_{2}\right)=d_{1}$ " for sure. This means that $P^{\beta}$ must be playing $c^{P O}$ with probability 1 at this equillibrium. But then $z^{\alpha}=1$ for sure which leads to the behavior " $e\left(\theta_{1}\right)=d_{1} ; e\left(\theta_{2}\right)=d_{2}$ " on the part of $A^{\alpha}$ in which case, however, it is optimal for $P^{\alpha}$ to play $c^{S E}$ with probability 1 rather than assigning any positive probability to $c^{P O}$. Thus, there cannot be any Principals' Equilibrium in this game.

Note that the crucial point here which leads to the absence of an equilibrium is that $z^{\beta}=2$ with positive probability if $P^{\alpha}$ plays $c^{P O}$ with positive probability, inducing $A^{\beta}$ to exhibit the undesirable behavior " $e\left(\theta_{1}\right)=d_{2} ; e\left(\theta_{2}\right)=d_{1}$ " for sure, no matter how small the probability $P^{\alpha}$ assigns to $c^{P O}$ is, since $A^{\beta}$ acts only knowing the probability distribution without having observed what contract actually gets realized. In our model, however, the fact that the agents move upon having perfectly observed the contracts chosen by the principals changes the matter entirely, leading to the existence of a zero - CE in our sense.

Here we consider the case where each $P^{i}$ plays $c^{S E}$ with probability $\lambda^{i}$ and $c^{P O}$ with probability $1-\lambda^{i}$, where $\lambda^{\alpha}=5 / 6$ and $\lambda^{\beta}=1 / 6$. To see that this strategy profile forms a $\mathrm{CE}$, first assume that $P^{\beta}$ chooses the contract randomization specified for her. Now, by choosing $c^{P O}, P^{\alpha}$ gets $V_{P}^{\alpha}\left(c^{P O},-\right)=5$ for sure, whereas, by choosing $c^{S E}$, she induces a probability distribution with two possible outcomes: There is a chance of 5/6 that $P^{\beta}$ chooses $c^{P O}$, whence $A^{\alpha}$ plays " $e\left(\theta_{1}\right)=d_{1} ; e\left(\theta_{2}\right)=d_{2}$ ", resulting in a payoff $V_{P}^{\alpha}\left(c^{S E}, c^{P O}\right)=6$ to $P^{\alpha}$. And there is a chance of $1 / 6$ that $P^{\beta}$ chooses $c^{S E}$, in which case $A^{\alpha}$ plays " $e\left(\theta_{1}\right)=d_{2}$; $e\left(\theta_{2}\right)=d_{1}$ ", and $P^{\alpha}$ gets $V_{P}^{\alpha}\left(c^{S E}, c^{S E}\right)=0$. Now the expected payoff $P^{\alpha}$ receives by playing $c^{S E}$ is given by $(5 / 6) 6+(1 / 6) 0=5$, and thus, she is indifferent between choosing $c^{S E}$ and $c^{P O}$. She is, therefore, prepared to play the contract combination specified for her if $P^{\beta}$ does so. Since a similar argument applies when the roles of $P^{\alpha}$ and $P^{\beta}$ are interchanged, the given contract combination forms a (zero) - CE. Note that, at this equilibrium, there is a chance of $5 / 36$ for the contract combination $\left(c^{S E}, c^{S E}\right)$ to occur under which $A^{\alpha}$ "cheats" $P^{\alpha}$. The 
contract profile where $P^{\alpha}$ plays $c^{P O}$ and $P^{\beta}$ chooses $c^{S E}$ and under which $A^{\beta}$ "cheats" $P^{\beta}$ occurs with a probability of $1 / 36$. In all the remaining combinations at which $P^{\beta}$ chooses $c^{P O}$ (with a probability of 5/6), both agents do what their principals want them to do.

The interaction of several principals in designing contracts for their agents has also been studied in the delegation literature by Fershtman and Judd (1987), Sklivas (1987), Koray and Sertel (1988, 1989), Katz (1991), and Cailland et al. (1995) among others. The main focus of these papers is on the strategic aspect of commitment effects achieved by letting a delegate (the agent) represent the main player (the principal) in some market or more generally in some normal form game as part of positive economics as well as from a regulatory viewpoint. Fershtman and Judd (1987), Sklivas (1987) and Koray and Sertel (1988) analyze the incentives created by the delegation mechanism for the principals to distort the maximands they hand down to their agents through publicly observable and irreversible contracts, and how these distortions affect the outcome of the agents' game. The first two papers interpret their results as providing an explanation for the compromise observed between sales- and profit-maximizing approaches, while the last paper employs the same construct for regulatory purposes, and Koray and Sertel (1989) questions the legitimacy of the positive approach adopted by Fershtman and Judd (1987) and Sklivas (1987). ${ }^{30}$ Katz (1991) provides a study of situations where delegation via unobservable contracts matters. Cailland et al. (1995) characterize economic situations where delegation via public contracts subject to renegotiation can have precommitment effects.

The main point in which the models in the delegation papers differ from that studied here concerns the underlying reason for the interdependence between different vertical structures: In the commitment literature, an interdependence between rivalrous principal-agent pairings arises because the profit accruing to one principal-agent structure depends not only on the action of the agent in that hierarchy, but also on that of the other agent. This "market interdependence" is absent in the present model. Our principals wish to condition their contracts on the decisions of each other's agents as well, only because these decisions provide information about their own agents' parameters about which the information is asymmetric. This "informational interdependence", on the other hand, is absent in the commitment literature. Other differences concern the restriction put on the space of admissible contracts, where the assumptions in the commitment literature range from very restrictive ${ }^{31}$ to very

\footnotetext{
${ }^{30}$ In the delegation mechanisms constructed in these papers, the maximand spaces, the delegation chain length and the solution concepts according to which the agents are instructed to resolve their game are all artificially restricted. It is shown in Koray and Sertel (1989) that self-interest-seeking principals have incentives not to obey these restrictions so long as there is no external rule forcing them to do so. The institution of possibly artificial rules to achieve efficiency, on the other hand, constitutes the essence of regulatory mechanism design.

${ }^{31}$ Fershtman and Judd (1987) and Sklivas (1987) restrict their attention to a setting in which the contracts in each agency can be based only on affine combinations of that agency's profits and sales a restriction which has been criticized by Koray and Sertel (1989), for the principals become strictly better off by not obeying this restriction.
} 
permissive $^{32}$ ones, and the way the issue of multiple equilibria in the agents' game is dealt with.

A more recent paper in the multiprincipals-multiagents literature is Martimort (1996). Martimort studies a setting of competing manufacturer-retailer hierarchies with adverse selection under the assumption of secret contracts. Like most of the papers discussed above, Martimort also focuses on truthful equilibria of incentive-compatible mechanisms ignoring the multiplicity problem. In his work, all choices made in a hierarchy are assumed to be unobservable by players in other hierarchies, so that any scope for yardstick competition between agents which is the main focus of the present paper - is eliminated.

\section{Conclusions}

In this paper we have studied the design of incentives through contractual arrangements in a model consisting of two ex ante identical principal-agent pairs. The notion of an Epsilon Contracting Equilibrium has been introduced to predict the outcome of the principals' contract design game, enabling us to appropriately deal with the discontinuities of the principals' payoff functions which arise when one passes the boundary between two domains of contract pairs inducing different agents' behaviors. It turns out that, in spite of the symmetric structure of the principals' game, there is no symmetric ECE in pure strategies. On the other hand, a symmetric ECE in mixed strategies exists and leads with positive probability to suboptimal contractual arrangements due to coordination failures among the principals.

The way we deal with the problem of multiplicity of equilibria here is through the introduction of a particular equilibrium selection criterion which consists of the concatenation of Payoff Dominance and Weak Firm Loyalty. A natural question which arises now is to what extent our results depend upon the selection criterion employed. We first note that, in the presence of multiple equilibria in the agents' game, each subgame perfect equilibrium of the entire game actually corresponds to a particular equilibrium selection. The materialization of each of these equilibria is again accompanied by the implicit assumption that the agents somehow coordinate their actions to reach that particular equilibrium and the principals share these equilibrium expectations when they make their own choices. Thus, focusing on any particular equilibrium selection gives rise to similar coordination problems along with the common knowledge problem about how these coordination problems are resolved.

In our context, some satisfactory solution to the problem of multiple equilibria is needed. It is true that there is some controversy about the employment of Payoff Dominance as an equilibrium selection criterion in spite of its intuitive appeal. Actually, the appropriateness of a selection criterion to deal with multiplicity

\footnotetext{
${ }^{32}$ In the 1987 version of his paper Katz (1991) allowed contracts in each agency to also depend on the contracts signed in the other agency. With such "cross-contingent-contracts" he showed that some kind of Folk Theorem could appear.
} 
of equilibria cannot be decided upon on a purely theoretical basis. This is an empirical, rather than a theoretical matter. What still can be done on a theoretical basis is, however, to discuss the robustness of our results here to alternative behavioral assumptions. Although this is an open problem yet to be solved, preliminary analysis in this direction suggests that other selection criteria will yield qualitatively similar results so long as the agents do not play the "worst" Bayesian equilibrium from their own viewpoint where this is common knowledge among all players.

Another question is to what extent the results of the present paper depend upon the structure of the space of admissible contracts. An assumption which is crucial in this regard for our results is that contracts cannot be conditioned on other contracts. The notion of contract, in general, can be thought of as covering a broad spectrum ranging from explicit agreements (as assumed in this paper) to implicit contracts (enforced through factors as firm-specific human capital, reputation, equilibrium unemployment, mobility costs) or to complicated mixed reward-punishment systems which include not only monetary components (as payments, stockholdings, dismissal related wealth consequences), but also nonmonetary factors (as power, prestige, honor). ${ }^{33}$ In the latter case, contracts based on the overall incentive structure in an industry are hard or impossible to verify even if "outside contracts" are observable ex post.

Regarding the structure of the contract spaces, there are two respects in which the contracts considered in the present paper differ from those on which standard implementation theory has usually focused in order to determine the optimal implementable benchmark: ${ }^{34}$ (i) Our agents" "verifiable reports" are not abstract but consist of real decisions; and (ii) the choice sets for the agents have too low a "dimension" to be adopted as message spaces in applying the Revelation Principle. To see the latter point, notice that an agent's type in a direct (revelation) mechanism is a summary of everything that he knows but which is either not common knowledge among all the players or unverifiable to outsiders. In the present context, an agent's relevant private or unverifiable information consists not only of the realization of the profitability variable which characterizes the technology under which he is employed, but also of the contract in the rival hierarchy. The message-space in a full-rank revelation scheme would, therefore, consist of reports in each of these dimensions. Allowing such two-dimensional reports would inevitably lead to a technical problem: Since there is no a-priori restriction on the set of admissible contracts and contracts might be conditioned

\footnotetext{
33 In a companion paper, Kerschbamer (1998) applies the model developed here to explain some seemingly paradoxical stylized facts from the takeover literature. There he identifies the contracts characterized in the present paper with categorical real world incentive systems. He argues (and presents evidence supporting this argument) that the incentives for managers generated by the compensation- and dismissal-policies of poorly motivated directors are similar to those created by weak comparative contracts: managers are penalized for underperforming in comparison to other firms in the industry; they are, however, not adequately rewarded for outperforming their rivals. The decision for a strong comparative contract is interpreted as a decision to motivate the board of directors.

34 That is, "direct revelation schemes" à la Myerson (1979) and Dasgupta et al. (1979).
} 
on contracts that are themselves conditioned on contracts ... there is no "natural" restriction on the sets of types. So the question arises whether it is possible to construct sets of types large enough to contain all the private and unverifiable information that agents might possibly have $\mathrm{e}^{35}$. Solving this problem and admitting full rank revelation schemes would mitigate the coordination problems discussed above; it would, however, provide no solution to the problem of multiple equilibria arising under a pair of weak comparative contracts. ${ }^{36}$ An even more complex message game, on the other hand, may suffice to stop agents from choosing undesired strategies, as work by Ma et al. (1988) and Mookherjee and Reichelstein (1990) suggests. The design of complex message games may be regarded as an elegant solution to the problem of multiple equilibria in a singleprincipal-multiple-agents context. In a multiple-principal context, however, the extreme coordination that such a design will require on the part of agents will render the materialization of its equilibria quite unrealistic.

The present work suggests a number of further avenues for future research: In our model the only motive for signing comparative contracts is the interdependence between the informational structures. If agencies are interpreted as firms other natural reasons for signing cross-contingent contracts come into one's mind: If firms interact in the same market, the market system will cause interdependences. Additional interdependences may arise if firms are linked by governmental interventions or by positive or negative externalities. The investigation of a setting with two or more sources for interdependences may yield interesting results. Another potentially fruitful area of research is collusion: The results in the present paper have been derived under the assumption that agents choose their strategies non-cooperatively. If collusion among agents cannot be precluded, the formal framework changes dramatically. In the limit - when agents behave as a single entity - we end up with a common agency with a two-dimensional uncertainty, an interesting framework from the viewpoint of design. ${ }^{37}$

Finally, we wish to note that the reason why we have introduced the notion of an Epsilon Contracting Equilibrium is not peculiar to our particular model, but is common to all principal-agent models where the induced behaviors of agents exhibit jump dicontinuities leading to dicontinuities of the same kind in the principals' utility functions. It is pretty clear that this phenomenon is typical of models where the agents' type spaces are finite or infinite but discrete, while the principals' choice sets form a continuum. In such models, the principals' joint

\footnotetext{
35 Besides this technical problem there is the question of whether it is sensible to assume that (verbal) reports (and not - as in the present context - only real decisions) in one hierarchy are verifiable by principals in rival hierarchies.

36 Allowing contracts where agents report types along the lines discussed above has similar consequences as admitting contract-contingent contracts. The main difference is that with unverifiable outside contracts a rent may be necessary to induce an agent to reveal his private or unverifiable information truthfully.

${ }^{37}$ In the one-dimensional case common agencies (i.e., scenarios in which one agent serves several principals) have been considered (among others) by Bernheim and Whinston (1986), Gal-Or (1991) and Martimort (1996). Single principal models in which the agent observes a multidimensional signal have, for example, been investigated by McAfee and McMillan (1988) and by Dana (1993).
} 
choice spaces are partitioned into regions on each of which the agents' induced actions stay constant. Now since optimality from the viewpoint of principals means inducing a desirable behavior on the part of agents through a minimal payout to these, seeking optimality drives principals to the boundary of the region corresponding to the agents' particular behavior they wish to induce. The point on the boundary towards which the principals converge, however, may not belong to the region itself and thus lead to different and undesirable actions. It is exactly this phenomenon which is formalized by the notion of Epsilon Contracting Equilibrium through which a whole family of principal-agent problems can be dealt with.

\section{Appendix 1}

This Appendix specifies the $V_{P}^{i}\left(c^{i}, c^{j}\right)$ 's for those contract combinations under which the Stage 1 participation constraint for at least one type of at least one agent is violated if this type expects the proposed continuation equilibrium at Stage 2. Nothing in the rest of the paper hinges on the exact form of these out-of-equilibrium-payoffs.

First consider those $c \in C$ for which $V_{A}^{i}\left(c \mid \theta_{h}^{i}\right)<0$ for all $\theta_{h}^{i} \in \Theta^{i}$ and $i \in$ $\{\alpha, \beta\}$. It is obvious that under these contract combinations participation of each type of each agent in the agents' game cannot be part of an overall equilibrium: Each type of each agent would have an incentive to reject the contract at Stage 1. The polar opposite strategy profile (both types of both agents reject the contract at Stage 1) cannot be part of an equilibrium either: One of the agents (say $A^{i}$ ) could obtain strict gains by accepting at Stage 1 and playing " $e^{i}\left(\theta_{1}^{i}\right)=$ $d_{1} ; e^{i}\left(\theta_{2}^{i}\right)=d_{2}$ " at Stage 2; since the payoff function in firm $i$ is given by " $g_{1}^{i}=\phi\left(\theta_{1}, d_{1}\right) ; g_{2}^{i}=\phi\left(\theta_{1}, d_{1}\right)+\phi\left(\theta_{2}, d_{2}\right)-\phi\left(\theta_{2}, d_{1}\right)$ " if firm $j$ is closed down (see Footnote 4$), A^{i}$, s interim payoffs would be $V_{A}^{i}\left(c \mid \theta_{1}^{i}\right)=0$ and $V_{A}^{i}\left(c \mid \theta_{2}^{i}\right)=$ $\phi\left(\theta_{2}, d_{1}\right)-\phi\left(\theta_{1}, d_{1}\right)>0$. We propose the following joint-behavior rule for these contract combinations: One of the firms (say firm $i$ ) is closed down and the agent in the second hierarchy (firm $j$ ) plays " $e^{j}\left(\theta_{1}^{j}\right)=d_{1} ; e^{j}\left(\theta_{2}^{j}\right)=d_{2}$ " as a best response to the payout function specified in Footnote 4. $P^{i}$ gets a payoff of 0 , $P^{j}$ gets $\lambda \equiv \phi\left(\theta_{1}, d_{1}\right)+\left(r_{21}+r_{22}\right)\left[\phi\left(\theta_{2}, d_{2}\right)-\phi\left(\theta_{2}, d_{1}\right)\right]$. The firm which is closed down is determined by a flip of a fair coin. Thus, the ex ante expected payoffs for the principals are: $V_{P}^{\alpha}\left(c^{\alpha}, c^{\beta}\right)=V_{P}^{\beta}\left(c^{\beta}, c^{\alpha}\right)=\lambda / 2$.

Next consider those $c \in C$ for which $V_{A}^{i}\left(c \mid \theta_{h}^{i}\right)<0$ for all $\theta_{h}^{i} \in \Theta^{i}$ and $V_{A}^{j}\left(c \mid \theta_{h}^{j}\right)>0$ for all $\theta_{h}^{j} \in \Theta^{j}$ where $\{i, j\}=\{\alpha, \beta\}$. Here we assume that firm $i$ is closed down and firm $j$ is operated under the payoff function specified in Footnote 4. Thus, $V_{P}^{i}\left(c^{i}, c^{j}\right)=0$ and $V_{P}^{j}\left(c^{j}, c^{i}\right)=\lambda$.

Finally consider those $c \in C$ for which $V_{A}^{i}\left(c \mid \theta_{h}^{i}\right) \geq 0$ for one type and $V_{A}^{i}\left(c \mid \theta_{h}^{i}\right)<0$ for the second type of at least one agent $i \in\{\alpha, \beta\}$. Here we propose that the whole procedure (calculation of a BE; checking whether all of the agents' interim-payoffs are nonnegative; if yes: calculation of the $V_{P}^{i}\left(c^{i}, c^{j}\right)$ 's; if not: repetition of the steps listed in this Appendix) is repeated for a modified 
agents' game in which types whose interim payoffs in the original agents' game were negative are removed and in which the agents' payoffs are calculated with the aid of the transfer function specified in Footnote 4.

\section{Appendix 2}

Case 3 of the Proof of the Nonexistence of a Symmetric Pure Strategy ECE:

We will treat this case by considering three subcases according as whether only $\theta_{2}$-agents randomize or only $\theta_{1}$-agents randomize or both kinds of agents randomize. For each $\epsilon \in(0, \bar{\epsilon}), i \in\{\alpha, \beta\}$ and $l \in\{1,2\}$, let $u_{l}^{i}(\epsilon)$ stand for the probability that $A^{i}$ plays $d_{l}$ upon observing $\theta_{l}$ at the agents' BE under $\left(\bar{c}^{\alpha}(\epsilon), \bar{c}^{\beta}(\epsilon)\right)$. Moreover, for each such $\epsilon, i$ and $k \in\{1,2\}$ set

$$
q_{k}^{i}(\epsilon)=R_{k 1} u_{1}^{j}(\epsilon)+R_{k 2}\left(1-u_{2}^{j}(\epsilon)\right),
$$

where $\{i, j\}=\{\alpha, \beta\}$. Note that $q_{k}^{i}(\epsilon)$ is nothing but the conditional probability that $A^{j}$ chooses $d_{1}$ given that $A^{i}$ has observed $\theta_{k}$ at the $\operatorname{BE}$ under $\left(\bar{c}^{\alpha}(\epsilon), \bar{c}^{\beta}(\epsilon)\right)$.

Subcase 1. First suppose that, for each $n \in \mathbf{N}$, there exists $\epsilon_{n} \in\left(0, \frac{1}{n}\right) \cap(0, \bar{\epsilon})$ such that the BE under $\left(\bar{c}^{\alpha}\left(\epsilon_{n}\right), \bar{c}^{\beta}\left(\epsilon_{n}\right)\right)$ is such that $u_{1}^{i}\left(\epsilon_{n}\right)=1$ and $u_{2}^{i}\left(\epsilon_{n}\right) \in(0,1)$ for $i \in\{\alpha, \beta\}$. Suppressing the superscript $i$, the sequences $\left(q_{1}\left(\epsilon_{n}\right)\right),\left(q_{2}\left(\epsilon_{n}\right)\right)$ and $\left(u_{2}\left(\epsilon_{n}\right)\right)$ are all bounded, so that they have convergent subsequences which we may without loss of generality assume to be already the sequences themselves. Now, for each $n \in \mathbf{N}$, set

$$
\begin{aligned}
I R_{1}^{\prime}\left(\epsilon_{n}\right) \quad & q_{1}\left(\epsilon_{n}\right) U\left(g_{11} \mid \theta_{1}\right)+\left(1-q_{1}\left(\epsilon_{n}\right)\right) U\left(g_{12} \mid \theta_{1}\right) \geq 0 \\
I R_{2}^{\prime}\left(\epsilon_{n}\right) \quad & u_{2}\left(\epsilon_{n}\right)\left[q_{2}\left(\epsilon_{n}\right) U\left(g_{21} \mid \theta_{2}\right)+\left(1-q_{2}\left(\epsilon_{n}\right)\right) U\left(g_{22} \mid \theta_{2}\right)\right] \\
& \left(1-u_{2}\left(\epsilon_{n}\right)\right)\left[q_{2}\left(\epsilon_{n}\right) U\left(g_{11} \mid \theta_{2}\right)+\left(1-q_{2}\left(\epsilon_{n}\right)\right) U\left(g_{12} \mid \theta_{2}\right)\right] \geq 0 \\
& q_{1}\left(\epsilon_{n}\right) U\left(g_{11} \mid \theta_{1}\right)+\left(1-q_{1}\left(\epsilon_{n}\right)\right) U\left(g_{12} \mid \theta_{1}\right) \geq \\
& q_{1}\left(\epsilon_{n}\right) U\left(g_{21} \mid \theta_{1}\right)+\left(1-q_{1}\left(\epsilon_{n}\right)\right) U\left(g_{22} \mid \theta_{1}\right) ; \\
S S_{2}^{\prime}\left(\epsilon_{n}\right) \quad & q_{2}\left(\epsilon_{n}\right) U\left(g_{21} \mid \theta_{2}\right)+\left(1-q_{2}\left(\epsilon_{n}\right)\right) U\left(g_{22} \mid \theta_{2}\right)= \\
& q_{2}\left(\epsilon_{n}\right) U\left(g_{11} \mid \theta_{2}\right)+\left(1-q_{2}\left(\epsilon_{n}\right)\right) U\left(g_{12} \mid \theta_{2}\right) ;
\end{aligned}
$$

and

$$
\begin{aligned}
v_{n}\left(g_{11}, g_{12}, g_{21}, g_{22}\right) & =\left(r_{11}+r_{12}\right)\left[q_{1}\left(\epsilon_{n}\right) g_{11}+\left(1-q_{1}\left(\epsilon_{n}\right)\right) g_{12}\right] \\
+ & \left(r_{21}+r_{22}\right)\left[u _ { 2 } ( \epsilon _ { n } ) \left(q_{2}\left(\epsilon_{n}\right) g_{21}+\left(1-q_{2}\left(\epsilon_{n}\right)\right) g_{22}\right.\right. \\
+ & \left(1-u_{2}\left(\epsilon_{n}\right)\right)\left(q_{2}\left(\epsilon_{n}\right) g_{11}+\left(1-q_{2}\left(\epsilon_{n}\right)\right) g_{12}\right] .
\end{aligned}
$$

Moreover, let $M W\left(\epsilon_{n}\right)$ stand for the program of maximizing $v_{n}$ subject to $I R_{1}^{\prime}\left(\epsilon_{n}\right)$, $I R_{2}^{\prime}\left(\epsilon_{n}\right), S S_{1}^{\prime}\left(\epsilon_{n}\right)$ and $S S_{2}^{\prime}\left(\epsilon_{n}\right)$. Now it is clear that $\left(\bar{g}_{11}\left(\epsilon_{n}\right), \bar{g}_{12}\left(\epsilon_{n}\right), \bar{g}_{21}\left(\epsilon_{n}\right), \bar{g}_{22}\left(\epsilon_{n}\right)\right)$ satisfies $I R_{1}^{\prime}\left(\epsilon_{n}\right), I R_{2}^{\prime}\left(\epsilon_{n}\right), S S_{1}^{\prime}\left(\epsilon_{n}\right), S S_{2}^{\prime}\left(\epsilon_{n}\right)$ for each $n \in \mathbf{N}$ by the choice of $\epsilon_{n}$.

Suppose that there is some sequence $\left(\delta_{n}\right)$ of positive real numbers with $\left(\delta_{n}\right) \rightarrow 0$ such that $\left|V_{P}\left(\bar{c}^{\alpha}\left(\epsilon_{n}\right), \bar{c}^{\beta}\left(\epsilon_{n}\right)\right)-v_{n}\left(g_{11}^{n}, g_{12}^{n}, g_{21}^{n}, g_{22}^{n}\right)\right|<\delta_{n}$, where 
$\left(g_{11}^{n}, g_{12}^{n}, g_{21}^{n}, g_{22}^{n}\right)$ stands for the solution of $M W\left(\epsilon_{n}\right)$ for each $n \in \mathbf{N}$. Denoting the limits of $\bar{g}_{k l}\left(\epsilon_{n}\right), q_{k}\left(\epsilon_{n}\right), u_{2}\left(\epsilon_{n}\right)$ as $n \rightarrow \infty$ by $\bar{g}_{k l}, \bar{q}_{k}, \bar{u}_{2}$, respectively, $(k, l \in\{1,2\})$, let $v_{\infty}, I R_{1}^{\prime}(0), I R_{2}^{\prime}(0), S S_{1}^{\prime}(0), S S_{2}^{\prime}(0)$ stand for the relations obtained from $v_{n}, I R_{1}^{\prime}\left(\epsilon_{n}\right), I R_{2}^{\prime}\left(\epsilon_{n}\right), S S_{1}^{\prime}\left(\epsilon_{n}\right), S S_{2}^{\prime}\left(\epsilon_{n}\right)$ by replacing $q_{k}\left(\epsilon_{n}\right), u_{2}\left(\epsilon_{n}\right)$ by $\bar{q}_{k}, \bar{u}_{2}$, respectively. Letting $M W(0)$ denote the corresponding program, it is seen that $\left(\bar{g}_{11}, \bar{g}_{12}, \bar{g}_{21}, \bar{g}_{22}\right)$ is a solution to $M W(0)$. Now it can be checked that $I R_{1}^{\prime}(0)$ is binding; $S S_{1}^{\prime}(0)$ holds with strict inequality, $\bar{g}_{11}<\bar{g}_{12}$ and $\bar{g}_{21}=\bar{g}_{22}$. Thus, in case $\bar{u}_{2}>0$, one has $\bar{q}_{2} \in(0,1)$, from which it follows that

$$
U\left(\bar{g}_{11} \mid \theta_{1}\right)>U\left(\bar{g}_{21} \mid \theta_{1}\right) \quad \text { and } \quad U\left(\bar{g}_{11} \mid \theta_{2}\right)>U\left(\bar{g}_{21} \mid \theta_{2}\right) \text {. }
$$

Then, however, by continuity of $U$ and the contracts,

$$
U\left(\bar{g}_{11}\left(\epsilon_{n}\right) \mid \theta_{1}\right)>U\left(\bar{g}_{21}\left(\epsilon_{n}\right) \mid \theta_{1}\right) \quad \text { and } \quad U\left(\bar{g}_{11}\left(\epsilon_{n}\right) \mid \theta_{2}\right)>U\left(\bar{g}_{21}\left(\epsilon_{n}\right) \mid \theta_{2}\right)
$$

for all sufficiently large $n$, in contradiction with $u_{2}\left(\epsilon_{n}\right) \in(0,1)$ because of Payoff Dominance. So, $\bar{u}_{2}=0$. Then, however, $\bar{q}_{1}=\bar{q}_{2}=1$, implying that $v_{\infty}\left(\bar{g}_{11}, \bar{g}_{12}, \bar{g}_{21}, \bar{g}_{22}\right)=\bar{g}_{11}<V_{P}\left(c^{I},-\right)$. Now this is easily seen to contradict that $\left(\bar{c}^{\alpha}\left(\epsilon_{n}\right), \bar{c}^{\beta}\left(\epsilon_{n}\right)\right)$ is an $\epsilon_{n}$-CE for sufficiently large $n$. Thus, there exist some $\delta>0$ and a subsequence of $\left(\epsilon_{n}\right)$ (which we may without loss of generality assume to be $\left(\epsilon_{n}\right)$ itself) such that $\left|V_{P}\left(\bar{c}^{\alpha}\left(\epsilon_{n}\right), \bar{c}^{\beta}\left(\epsilon_{n}\right)\right)-v_{n}\left(g_{11}^{n}, g_{12}^{n}, g_{21}^{n}, g_{22}^{n}\right)\right| \geq \delta$ for all $n \in \mathbf{N}$. But this simply means that $\left(a_{2}\left(\epsilon_{n}\right)\right): U\left(\bar{g}_{21}\left(\epsilon_{n}\right) \mid \theta_{2}\right)>U\left(\bar{g}_{11}\left(\epsilon_{n}\right) \mid \theta_{2}\right)$ holds for all $n \in \mathbf{N}$.

But then $v_{n}\left(g_{11}^{n}, g_{12}^{n}, g_{21}^{n}, g_{22}^{n}\right) \geq V_{P}\left(\bar{c}^{\alpha}\left(\epsilon_{n}\right), \bar{c}^{\beta}\left(\epsilon_{n}\right)\right)+\delta$ for all $n \in \mathbf{N}$. Now, for each $n \in \mathbf{N}$ with $\epsilon_{n}<\delta$, if $c^{\prime \alpha} \in C^{\alpha}$ is a contract whose payoff schedule is given by $\left(g_{11}^{n}, g_{12}^{n}, g_{21}^{n}, g_{22}^{n}\right)$, then $V_{P}^{\alpha}\left(c^{\prime \alpha}, \bar{c}^{\beta}\left(\epsilon_{n}\right)\right)=v_{n}\left(g_{11}^{n}, g_{12}^{n}, g_{21}^{n}, g_{22}^{n}\right)>$ $V_{P}^{\alpha}\left(\bar{c}^{\alpha}\left(\epsilon_{n}\right), \bar{c}^{\beta}\left(\epsilon_{n}\right)\right)+\epsilon_{n}$, a contradiction. Therefore, there is some $\tilde{\epsilon}>0$ with $\tilde{\epsilon} \leq \bar{\epsilon}$ such that there is no (symmetric) BE under $\left(\bar{c}^{\alpha}(\epsilon), \bar{c}^{\beta}(\epsilon)\right)$ with $u_{1}(\epsilon)=1$, $u_{2}(\epsilon) \in(0,1)$ whenever $\epsilon \in(0, \tilde{\epsilon})$.

Subcase 2. Now suppose that, for each $n \in \mathbf{N}$, there exists $\epsilon_{n} \in\left(0, \frac{1}{n}\right) \cap(0, \bar{\epsilon})$ such that one has $u_{1}\left(\epsilon_{n}\right) \in(0,1)$ and $u_{2}\left(\epsilon_{n}\right)=1$ at the BE under $\left(\bar{c}^{\alpha}\left(\epsilon_{n}\right), \bar{c}^{\beta}\left(\epsilon_{n}\right)\right)$. We make similar observations as in the above subcase regarding convergence of sequences here and will use a parallel notation.

For each $n \in \mathbf{N}$, set

$$
\begin{array}{cl}
\left(I R_{1}^{\prime \prime}\left(\epsilon_{n}\right)\right) & u_{1}\left(\epsilon_{n}\right)\left[q_{1}\left(\epsilon_{n}\right) U\left(g_{11}\left(\epsilon_{n}\right) \mid \theta_{1}\right)+\left(1-q_{1}\left(\epsilon_{n}\right)\right) U\left(g_{12}\left(\epsilon_{n}\right) \mid \theta_{1}\right)\right] \\
+(1- & \left.u_{1}\left(\epsilon_{n}\right)\right)\left[q_{1}\left(\epsilon_{n}\right) U\left(g_{21}\left(\epsilon_{n}\right) \mid \theta_{1}\right)+\left(1-q_{1}\left(\epsilon_{n}\right)\right) U\left(g_{22}\left(\epsilon_{n}\right) \mid \theta_{1}\right)\right] \geq 0 ; \\
\left(I R_{2}^{\prime \prime}\left(\epsilon_{n}\right)\right) \quad & q_{2}\left(\epsilon_{n}\right) U\left(g_{21}\left(\epsilon_{n}\right) \mid \theta_{2}\right)+\left(1-q_{2}\left(\epsilon_{n}\right)\right) U\left(g_{22}\left(\epsilon_{n}\right) \mid \theta_{2}\right) \geq 0 ; \\
\left(S S_{1}^{\prime \prime}\left(\epsilon_{n}\right)\right) \quad & q_{1}\left(\epsilon_{n}\right) U\left(g_{11}\left(\epsilon_{n}\right) \mid \theta_{1}\right)+\left(1-q_{1}\left(\epsilon_{n}\right)\right) U\left(g_{12}\left(\epsilon_{n}\right) \mid \theta_{1}\right) \\
= & q_{1}\left(\epsilon_{n}\right) U\left(g_{21}\left(\epsilon_{n}\right) \mid \theta_{1}\right)+\left(1-q_{1}\left(\epsilon_{n}\right)\right) U\left(g_{22}\left(\epsilon_{n}\right) \mid \theta_{1}\right) ; \\
\left(S S_{2}^{\prime \prime}\left(\epsilon_{n}\right)\right) \quad & q_{2}\left(\epsilon_{n}\right) U\left(g_{21}\left(\epsilon_{n}\right) \mid \theta_{2}\right)+\left(1-q_{2}\left(\epsilon_{n}\right)\right) U\left(g_{22}\left(\epsilon_{n}\right) \mid \theta_{2}\right) \\
\geq & q_{2}\left(\epsilon_{n}\right) U\left(g_{11}\left(\epsilon_{n}\right) \mid \theta_{2}\right)+\left(1-q_{2}\left(\epsilon_{n}\right)\right) U\left(g_{12}\left(\epsilon_{n}\right) \mid \theta_{2}\right) ;
\end{array}
$$

and 


$$
\begin{aligned}
v_{n}\left(g_{11}, g_{12}, g_{21}, g_{22}\right) & =\left(r_{11}+r_{12}\right)\left[u_{1}\left(\epsilon_{n}\right)\left(q_{1}\left(\epsilon_{n}\right) g_{11}+\left(1-q_{1}\left(\epsilon_{n}\right)\right) g_{12}\right)\right. \\
+ & \left.\left(1-u_{1}\left(\epsilon_{n}\right)\right)\left(q_{1}\left(\epsilon_{n}\right) g_{21}+\left(1-q_{1}\left(\epsilon_{n}\right)\right) g_{22}\right)\right] \\
+ & \left(r_{21}+r_{22}\right)\left(q_{2}\left(\epsilon_{n}\right) g_{21}+\left(1-q_{2}\left(\epsilon_{n}\right)\right) g_{22}\right) .
\end{aligned}
$$

We denote the corresponding program by $M W\left(\epsilon_{n}\right)$ and its solution by $\left(g_{11}^{n}, g_{12}^{n}, g_{21}^{n}\right.$, $\left.g_{22}^{n}\right)$. The same notation as in subcase 1 is used for the limiting constraints, the objective function and the resulting program.

Under the corresponding supposition about the existence of a sequence $\left(\delta_{n}\right)$ of positive real numbers with $\left(\delta_{n}\right) \rightarrow 0$ and $\mid V_{P}\left(\bar{c}^{\alpha}\left(\epsilon_{n}\right), \bar{c}^{\beta}\left(\epsilon_{n}\right)\right)-v_{n}\left(g_{11}^{n}, g_{12}^{n}, g_{21}^{n}\right.$, $\left.g_{22}^{n}\right) \mid<\delta_{n}$, it is seen that $\left(\bar{g}_{11}, \bar{g}_{12}, \bar{g}_{21}, \bar{g}_{22}\right)$ is a solution to $M W(0)$. Now it can be checked that $I R_{2}^{\prime \prime}(0)$ is binding, $S S_{2}^{\prime \prime}(0)$ holds with strict inequality, $\bar{g}_{11}=\bar{g}_{12}$ and $\bar{g}_{22}<\bar{g}_{21}$. The rest of the proof can now be obtained from subcase 1 by interchanging the roles of $\left(\theta_{1}, d_{1}\right)$ and $\left(\theta_{2}, d_{2}\right)$.

Subcase 3. We finally suppose that, for each $n \in \mathbf{N}$, there is some $\epsilon_{n} \in$ $\left(0, \frac{1}{n}\right) \cap(0, \bar{\epsilon})$ such that one has $u_{1}\left(\epsilon_{n}\right), u_{2}\left(\epsilon_{n}\right) \in(0,1)$ at the agents' BE. Then $S S_{1}^{\prime \prime}\left(\epsilon_{n}\right)$ and $S S_{2}^{\prime \prime}\left(\epsilon_{n}\right)$ both must be satisfied for each $n \in \mathbf{N}$. It can now easily be checked that $\phi\left(\theta_{1}, d_{2}\right)-\bar{g}_{22}\left(\epsilon_{n}\right) \neq \phi\left(\theta_{1}, d_{1}\right)-\bar{g}_{12}\left(\epsilon_{n}\right)$. Now consider the following four cases:

$$
\begin{aligned}
& \phi\left(\theta_{1}, d_{2}\right)-\bar{g}_{22}\left(\epsilon_{n}\right)>\phi\left(\theta_{1}, d_{1}\right)-\bar{g}_{12}\left(\epsilon_{n}\right) \quad \text { and } \quad \bar{g}_{21}\left(\epsilon_{n}\right) \leq \bar{g}_{22}\left(\epsilon_{n}\right) ; \\
& \begin{array}{rrrr}
\text { (iii) } \phi\left(\theta_{1}, d_{2}\right)-\bar{g}_{22}\left(\epsilon_{n}\right)<\phi\left(\theta_{1}, d_{1}\right)-\bar{g}_{12}\left(\epsilon_{n}\right) & \text { and } & \bar{g}_{11}\left(\epsilon_{n}\right)>\bar{g}_{12}\left(\epsilon_{n}\right) \text {; } \\
\text { (iv }) & \# & \text { and } & \bar{g}_{11}\left(\epsilon_{n}\right) \leq \bar{g}_{12}\left(\epsilon_{n}\right) \text {. }
\end{array}
\end{aligned}
$$

Now there are a case $(j)$ from among $(i)-(i v)$ above and a subsequence $\left(\epsilon_{n_{k}}\right)$ of $\left(\epsilon_{n}\right)$ such that case $(j)$ is satisfied for all $k \in \mathbf{N}$. Again without loss of generality, one can assume the subsequence $\left(\epsilon_{n_{k}}\right)$ to be $\left(\epsilon_{n}\right)$ itself. Finally, it can be checked that each of the cases $(i)-(i v)$ implies that either $\bar{u}_{1}$ or $\bar{u}_{2} \in\{0,1\}$ which leads to the desired contradiction along similar lines as in subcase 1 or 2 above.

\section{References}

Aumann, R., Katznelson, Y., Radner, R., Rosenthal, R., Weiss, B. (1983) Approximate purification of mixed strategies. Mathematics of Operations Research 8: 327-341

Berge, C. (1963) Topological Spaces. MacMillan, New York

D., Bernheim, M. Whinston (1986) Common agency. Econometrica 54: 923S-942

Cailland, B., Jullien, B., Picard, P. (1995) Competing vertical structures: Precommitment and renegotiation. Econometrica 63: 621-646

Dana, J. (1993) The organization and scope of agents: Regulating multiproduct industries. Journal of Economic Theory 59: 288-310

Dasgupta, P., Hammond, P., Maskin, E. (1979) The implementation of social choice rules: Some general results on incentive compatibility. The Review of Economic Studies 46: 185-216

Demski, J., Sappington, D., (1984) Optimal incentives with multiple agents. Journal of Economic Theory 33: 152-171

Fershtman, C., Judd, K., (1987) Equilibrium incentives in oligopoly. American Economic Review 77: 927-940

Gal-or, E. (1991) A common agency with incomplete information. Rand Journal of Economics 22: 274-286 
Harsanyi, J. (1973) Games with randomly disturbed payoffs: A new rationale for mixed strategy equilibrium points. International Journal of Game Theory 2: 1-23

Katz, M. (1991) Game-playing agents: Unobservable contracts as precommitments. Rand Journal of Economics 23: 307-328

Kerschbamer, R. (1994) Destroying the 'pretending' equilibrium in the Demski-Sappington-Spiller model. Journal of Economic Theory 62: 230-237

Kerschbamer, R. (1998) Disciplinary takeovers and industry effects. Journal of Economics and Management Strategy 7: 265-306

Koray, S. Sertel, M.R. (1988) Regulating a duopoly by a pretend-but-perform mechanism. In: Holler, M., Rees, R. (eds.): Economics of Market Structure. Special issue of the European Journal of Political Economy 4: 95-115

Koray, S., Sertel, M.R. (1989) Meta-Cournotic equilibrium in oligopoly: Positive or regulatory theory? Working Paper Nr 89-01-02, Risk and Decision Processes Center, The Wharton School of the University of Pennsylvania

Ma, C., Moore, J., Turnbull, S. (1988) Stopping agents from cheating. Journal of Economic Theory 46: $355-372$

Martimort, D. (1996) Exclusive dealing, common agency, and multiprincipals incentive theory. Rand Journal of Economics 27: 1-31

McAfee, P., McMillian, J. (1988) Multidimensional incentive compatibility and mechanism design. Journal of Economic Theory 46: 335-354

Mookherjee, D. (1984) Optimal incentive schemes with many agents. Review of Economic Studies 51: 433-446

Mookherjee, D., Reichelstein, S. (1990) Implementation via augmented revelation mechanisms. Review of Economic Studies 57: 453-475

Myerson, R. (1979) Incentive compatibility and the bargaining problem. Econometrica 47: 61-73

Myerson, R. (1982) Optimal coordination mechanisms in generalized principal-agent problems. Journal of Mathematical Economics 10: 67-81

Radner, R. (1980) Collusive behavior in oligopolies with long but finite lives. Journal of Economic Theory 22: $136-156$

Sklivas, S. (1987) The strategic choice of managerical incentives. Rand Journal of Economics 18: $452-458$ 